\title{
The Spectral Properties of the Two Rod
}

\section{Pathways}

\author{
LINDSAY T. SHARPE,* CLEMENS C. FACH,* ANDREW STOCKMAN $\dagger$ \\ Received 27 August 1992; in revised form 26 April 1993
}

\begin{abstract}
Psychophysical and electroretinographic observations in normal and achromat observers suggest that rod flicker signals have access to at least two retinal pathways: one $\left(\pi_{0}\right)$, slow and sensitive, predominating at scotopic luminance levels; the other $\left(\pi_{0}^{\prime}\right)$, fast and insensitive, predominating at mesopic ones. We have measured steady-state flicker detection sensitivities on background fields ranging from 430 to $640 \mathrm{~nm}$ in normal observers. Our results suggest that cone signals can reduce the sensitivity of $\pi_{0}^{\prime}$, but have comparatively little effect on $\pi_{0}$. The $\pi_{0}^{\prime}$ field sensitivities derived from these measurements have been fitted with linear combinations of the scotopic luminosity function, $V_{\lambda}^{\prime}$, the $M$-cone spectral sensitivity function, $M_{\lambda}$, and the $L$-cone function, $L_{\lambda}$. These fits demonstrate a clear cone influence on $\pi_{0}^{\prime}$, but they cannot tell us unequivocally whether the influence is from the M-cones, from the L-cones or from both. Accordingly, we made similar measurements in dichromats, who lack one of the two longer wavelength cone types. These measurements revealed an L-cone influence on $\pi_{0}^{\prime}$ in the deuteranope and an $M$-cone influence in the protanope. This suggests that both cone types can affect the sensitivity of $\pi_{0}^{\prime}$. The finding that in the steady-state cone signals reduce the sensitivity of $\pi_{0}^{\prime}$ but have little effect on $\pi_{0}$ could suggest that $\pi_{0}^{\prime}$ signals travel through a faster cone pathway (with its own gain control at which both rod and cone signals can reduce rod threshold), while $\pi_{0}$ signals travel through a separate rod pathway. However, it could simply reflect the fact that $\pi_{0}^{\prime}$ predominates at higher luminances than $\pi_{0}$ where the cone excitation level is inevitably greater. To examine the influence of the cones on $\pi_{0}$ more closely, we: (i) produced transient cone excitation by alternating rod-equated 480 and $679 \mathrm{~nm}$ fields; and (ii) extended our steady-state measurements to include deep-red backgrounds of 650 and $680 \mathrm{~nm}$. Both experiments revealed a small, but measurable influence of the cones on $\pi_{0}$.
\end{abstract}

Rods Temporal sensitivity Flicker Spectral sensitivity Field sensitivity Rod-cone interaction

\section{INTRODUCTION}

Psychophysical data (Conner \& MacLeod, 1977; Conner, 1982; Sharpe, Stockman \& MacLeod, 1989) and electroretinographic (ERG) recordings (Stockman, Sharpe, Zrenner \& Nordby, 1991) in the normal and achromat observer demonstrate that rod flicker signals have access to both a slow and a fast retinal pathway. The slower pathway [labeled $\pi_{0}$, after the notation of Stiles (1978)] is more sensitive and dominates from absolute threshold up to low mesopic levels. The faster pathway (labeled $\pi_{0}^{\prime}$ ) is less sensitive and takes over flicker detection only at moderate and high mesopic levels.

The two pathways are most clearly revealed in the double-branching of scotopic critical flicker fusion (CFF) vs intensity functions (Hecht, Shlaer, Smith, Haig \& Peskin, 1938; Hecht, Shlaer, Smith, Haig \& Peskin, 1948; Conner \& MacLeod, 1977; Hess \&

*Neurologische Universitätsklinik, Hansastraße 9, D-9104 Freiburg i. Br., Germany.

†Department of Psychology, University of California at San Diego, La Jolla, CA 92093-0109, U.S.A.
Nordby, 1986a) and scotopic flicker threshold vs intensity (TVI) profiles measured with frequencies $>8 \mathrm{~Hz}$ (Conner, 1982; Sharpe et al., 1989). Al or near $15 \mathrm{~Hz}$, there is a suprathreshold intensity region, adjoining the double-branched flicker TVI function, within which the perception of flicker completely disappears (see Fig. 1). Counterintuitively, therefore, increasing the intensity of $15 \mathrm{~Hz}$ flicker causes the flicker to disappear before reappearing again at higher intensities. This cancellation or null can be explained by destructive interference between slow $\pi_{0}$ rod signals, and fast $\pi_{0}^{\prime}$ rod signals, which are approx. $180 \mathrm{deg}$ out of phase with one another near $15 \mathrm{~Hz}$ (Sharpe et al., 1989). To explain the restricted range of the null, we assume that the intensity dependencies of $\pi_{0}$ and $\pi_{0}^{\prime}$ differ, such that the former predominates at intensities below the null and the latter at intensities above the null; the two being approximately equal at intensities within the null (Conner, 1982; Sharpe et al., 1989; Sharpe \& Stockman, 1991; Stockman et al., 1991).

Though it can be easily demonstrated that flicker detection on each branch of the flicker TVI or CFF curves is mediated by rods (Conner \& MacLeod, 1977; Conner, 1982; Sharpe et al., 1989; Stockman et al., 
1991), the regulation of sensitivity on each branch may depend on both rods and cones. Indeed, for other detection tasks there is ample evidence that the cones can decrease rod sensitivity, particularly at high light levels (e.g. Makous \& Boothe, 1974; Sternheim \& Glass, 1975; Frumkes \& Temme, 1977; Sharpe, Fach, Nordby \& Stockman, 1989).

One way of investigating the spectral properties of the sensitivity regulating mechanisms underlying $\pi_{0}$ and $\pi_{0}^{\prime}$ is to determine their field sensitivities (see Stiles, 1978). A field sensitivity is the reciprocal of the field intensity required to raise rod flicker threshold by some criterion amount, measured as a function of field wavelength. If the regulation of rod flicker sensitivity depends only on rods, the field sensitivity will be a scotopic $\left(V_{\lambda}^{\prime}\right)$ spectral sensitivity; but if cones also regulate rod flicker sensitivity, the field sensitivity will deviate from $V_{i}^{\prime}$-most likely at long wavelengths, where the cones, relative to the rods, are most sensitive. Two studies have looked at this question before, but with conflicting results. Conner (1982) claimed to find no cone influence on either $\pi_{0}$ or $\pi_{0}^{\prime} ;$ whereas Knight, Sanocki and Buck (1990) claimed to find a clear cone influence on $\pi_{0}^{\prime}$, but not on $\pi_{0}$ (see below).

If the cones do decrease rod flicker sensitivity, are they likely to have a larger effect on $\pi_{0}^{\prime}$ than on $\pi_{0}$ ? Perhaps the simplest model is one in which the cone influence on rod flicker sensitivity rises with the level of cone excitation, regardless of whether flicker detection is mediated by $\pi_{0}$ or $\pi_{0}^{\prime}$. This model predicts that cone signals are less likely to modify the flicker sensitivity of $\pi_{0}$ than $\pi_{0}^{\prime}$, merely because $\pi_{0}$ predominates at intensity levels where the cones are relatively unadapted, and it predicts a fairly gradual increase in cone influence on both $\pi_{0}$ or $\pi_{0}^{\prime}$ as the background luminance increases to mesopic levels.

Another plausible model of the rod flicker duality is that $\pi_{0}^{\prime}$ reflects the transmission of rod signals through faster retinal pathways that are intended primarily for cones, whereas $\pi_{0}$ reflects their transmission through more sluggish rod pathways. In the cat, the mammalian species for which we have some of the most detailed information about retinal function, the fast pathway might be from the rods to the cones via the rod-cone gap junctions, to the cone bipolars, and then to the $O N$ and OFF ganglion cells; and the slow pathway might be from the rods to the rod bipolars, to the AII amacrine cells, and then to either $\mathrm{ON}$ cone bipolars and $\mathrm{ON}$ ganglion cells or to OFF ganglion cells (see Daw, Jensen \& Brunken, 1990 for a review; Sterling, 1983; Sterling, Freed \& Smith, 1986). According to this model, like the previous one, cone signals are more likely to modify the flicker sensitivity of $\pi_{0}^{\prime}$ and $\pi_{0}$. In this case, however, we should expect the field sensitivity to change abruptly as detection passes from $\pi_{0}$ to $\pi_{0}^{\prime}$.

Previous field sensitivity measurements for the detection of flashes show that at low rod threshold elevations (i.e. when the background intensity is scotopic) field sensitivity is purely rod-determined, while at high elevations (i.e. when the background intensity is mesopic) it is determined by both the rods and cones (e.g. Flamant \& Stiles, 1948; Makous \& Boothe, 1974; Makous \& Peeples, 1979; Sharpe et al., 1989). This result could be consistent with the hypothesis that cone influence grows with the level of cone excitation regardless of the pathway, but it could also be consistent with the hypothesis that $\pi_{0}^{\prime}$ is differentially affected by cones, since flash detection-like flicker detection - may pass from $\pi_{0}$ to $\pi_{0}^{\prime}$ as the intensity is increased (a possibility suggested to us by S. L. Buck, personal communication).

If the field sensitivity for flicker detection on either branch of the flicker TVI function deviates from $V_{\lambda}^{\prime}$, the way in which it does so should reveal something about the nature of the underlying sensitivity regulating mechanisms. In particular, it might show whether the $\mathrm{M}$ - or the $\mathrm{L}$-cones are primarily involved in the regulation of rod flicker sensitivity. Flash detection measurements of spectral sensitivity demonstrate the L-cone influence more clearly (e.g. Alexander \& Kelly, 1984; Alexander, Kelly \& Morris, 1986; Sharpe, Fach \& Stockman, 1992), presumably because the L-cones are more sensitive at long wavelengths, where the cone influence on rods is most easily measured. Yet, if transient cone excitation is used, a clear M-cone influence on rod flash detection can be revealed (Sharpe et al., 1992). In rod flicker detection experiments, Knight, Buck and Sanocki (1991) claimed to find a clear effect of M-cones on the sensitivity on $\pi_{0}^{\prime}$, but little or no effect of L-cones. This result is surprising and contradicts the results of the flash detection experiments. In this paper, we measured $\pi_{0}^{\prime}$ field sensitivities in a normal, a deuteranope and a protanope in order to reveal the contribution of the $\mathrm{M}$ - and L-cones to the elevation of the rod flicker threshold.

\section{METHODS}

\section{Subjects}

Four normal trichromats, CF, RT, JH and LTS, a deuteranope, NE, a protanope, JS, and an achromat, $\mathrm{KN}$, served as observers in this study. Three of the normal observers, CF, RT and JH are emmetropic. LTS is slightly myopic $(-2 \mathrm{D})$, but required no corrective lenses. All four have normal color vision as indicated by the conventional acuity and color vision tests. The deuteranope and the protanope are emmetropic. Their color vision deficiency was diagnosed by the Ishihara pseudoisochromatic plates, the Rayleigh equation on the Nagel anomaloscope and the Farnsworth-Munsell 100Hue test. The achromat observer displays all the classic symptoms of typical, complete achromatopsia (see Sharpe \& Nordby, 1990 for a full description); and extensive physiological testing reveals no residual cone function (Nordby, Stabell \& Stabell, 1984; Hess \& Nordby, 1986a, b; Hess, Nordby \& Pointer, 1987; Nordby \& Sharpe, 1988; Sharpe, van Norren \& Nordby, 1988). He is hyperopic and during the experiments wore corrective spectacles $(+9 \mathrm{D})$ which magnified the retinal image approx. 1.22 times. 


\section{Apparatus and stimuli}

A three-channel Maxwellian-view stimulator (see also Nordby \& Sharpe, 1988; Sharpe \& Volbrecht, 1990) produced the flickering test stimuli, the steady background field and the bleaching light. All three channels originated from a $100 \mathrm{~W}$ tungsten-iodine lamp, run at constant current. One channel provided the $6 \mathrm{deg}$ dia flickering test light. Its wavelength was shaped by a grating monochromator (Jobin-Yvon $\mathrm{H}-10 \mathrm{Vis}$ ) into a triangular profile, peaking at $500 \mathrm{~nm}$ and having a bandwidth of $4 \mathrm{~nm}$. A second channel provided a $18 \mathrm{deg}$ dia background. It was rendered monochromatic by interference filters (Schott). The third channel provided the $20 \mathrm{deg}$ dia, white $(3100 \mathrm{~K})$ bleaching light. The target, the background field and the bleach were centered $12 \mathrm{deg}$ from the fovea in the nasal field of view. Fixation was aided by an illuminated red cross.

Except for the achromat, the entry point of the flickering target was offset in the pupil by $3 \mathrm{~mm}$ from the pupillary center to favor rod detection of the flicker. The entry point of the adapting field was central.

Small circular aperture stops, placed at the focal points of the filament lamp in each channel, restricted the filament images to $<2 \mathrm{~mm}$ in diameter at the plane of the observer's pupil. Circular field stops placed in collimated beams in each channel defined the test and background fields as seen by the observer. Electromagnetic shutters were positioned in each channel near focal points of the filament lamp. The shutter in the test channel was run by a shutter driver connected to a frequency generator (Wavetek). With this arrangement we could obtain reliable square waves at the target frequencies used: $8,11,14$ and $15 \mathrm{~Hz}$. Given the insensitivity of the rod visual system to high frequency flicker, the weaker, higher sinusoidal harmonics of the square waves (at $3 \times, 5 \times, 7 \times$ the fundamental frequency, etc.) should have little effect on our threshold measurements. The optical waveforms so produced were monitored periodically using a Pin-10 photodiode (United Detector Technology) and oscilloscope. In some additional experiments (see Results, last section), sinusoidal, instead of square wave, flicker at 8 and $15 \mathrm{~Hz}$ was used. The sinusoidal flicker was produced by pulse width modulating ferroelectric liquid crystal shutters (LVA-0.7-VIS Light Valve, Displaytech Inc., Boulder, Colo.)

Fine control over the luminance of the stimuli was achieved by variable, circular neutral density wedges (Barr \& Stroud, Annisland), positioned close to image points of the filament lamp, and by the insertion of fixed neutral density filters in parallel portions of the beams.

The radiant fluxes of the test and adapting field stimuli were measured at the plane of the observer's entrance pupil with a calibrated radiometer/photometer (United Detector Technology, Model 360 Optometer).

\section{Procedure}

Before beginning each experimental session, the observer had his left pupil dilated with $0.5 \%$ tropicamide (Mydriaticum Roche ${ }^{\circledR}$ ). Following $\mathbf{4 0}$ min of dark adap- tation, he positioned himself in the optical system by biting into a silicone-base, dental-wax impression of his teeth mounted in a machine tool-rest, and fixated the illuminated cross. The intensity of the square wave flickering rod stimulus required just to detect flicker was determined using a computer-controlled, single-staircase procedure (Cornsweet, 1962), with a step size of $0.04 \mathrm{log}$ unit in intensity. Threshold was defined as the mean of 12 staircase reversals. In a single session, thresholds were measured on a series of adapting fields of increasing intensity. At each new intensity level the observer adapted for at least $3 \mathrm{~min}$ before measuring the threshold.

After completing the flicker threshold settings at several background intensities, the observer determined, in a separate run, the limits of the null region. The intensities of the suprathreshold rod stimulus were found at which the sensation of flicker first vanished and then reappeared (this was only possible for frequencies near $15 \mathrm{~Hz}$ ). These settings repeated 12 times, defined the lower and upper limits of the null region.

Cone thresholds were obtained by making measurements during the plateau that terminates the cone phase of recovery from a white $(3100 \mathrm{~K})$ bleaching light of $7.7 \log$ phot. td sec (i.e. between 6 and 10 min following the end of the bleach).

\section{Threshold vs intensity curves and field sensitivities}

To investigate the spectral properties of the sensitivity regulating mechanisms underlying $\pi_{0}$ and $\pi_{0}^{\prime}$, we used the field sensitivity method of W. S. Stiles (see Stiles, 1978 for a compilation of his papers). In the field sensitivity method, TVI functions are measured at a series of field wavelengths $(\mu)$. The field sensitivity is then defined as the reciprocal of the field intensity at each $\mu$ required to raise threshold by some criterion amount. By measuring complete TVI functions, it is possible to determine whether the field spectral sensitivity changes as the criterion threshold rises. If it does not, the shapes of the TVI functions are invariant with changes in $\mu$ and Stiles' field displacement rule is said to hold. Shape invariance and a $V_{\lambda}^{\prime}$ field spectral sensitivity are to be expected if the rods alone determine sensitivity (see e.g. Stiles, 1978, for further discussion).

We determined the field spectral sensitivity of the lower, $\pi_{0}$ branches of the rod flicker TVI curves (which correspond approximately to field illuminances of -4.0 to $-0.5 \log$ scot td) by fitting the threshold data with a standard template shape. This technique was feasible for the $\pi_{0}$ branch, because its shape is roughly invariant with changes in $\mu$ (but see Figs 15-17). The analysis was done separately for each subject (CF, NE and JS) and for each flicker frequency $(8,11$ or $15 \mathrm{~Hz})$. To derive the standard template shapes, we first averaged the TVI data across all $\mu$. The templates were then estimated by fitting a second-order polynomial to the mean data. All fits were carried out by means of a standard curve fitting program (Multigraf, Weka Software GmbH, Frankfurt, Germany). At 11 and $15 \mathrm{~Hz}$, the final part of the lower branch (where, according to our model, $\pi_{0}$ and $\pi_{0}^{\prime}$ 
destructively interfere) was too steep to be described by a second-order polynomial. A simple straight line segment was used to steepen and to extend the polynomial into that region. Having derived a standard template for each subject, we then fitted it to the individual data for each field wavelength by shifting the templatc along the abscissa to find the horizontal position where the sum of the squared deviations was least. (Small shifts along the ordinate were also allowed to account for day-to-day shifts in sensitivity.) The position of the template was then used to provide an estimate of the field sensitivity of that branch.

A similar method could not be used to estimate the field sensitivities of the upper, $\pi_{0}^{\prime}$ branch (which corresponds approximately to field illuminances of $-0.5 \mathrm{log}$ scot td to the cone threshold), because the slope of the upper branch steepens at long wavelengths $(\mu \geqslant 602 \mathrm{~nm})$. Therefore, an average template shape (derived as for the lower branch but only for $\mu<602 \mathrm{~nm}$ ) was fitted to the data points on the upper branch for $\mu<602 \mathrm{~nm}$. At $\mu \geqslant 602 \mathrm{~nm}$, we fitted secondorder polynomials to the individual data points for each $\mu$. In all cases, the field points, corresponding to the criterion threshold elevation (see below), were then read off either the fitted template or the fitted polynomial. The fact that a standard template cannot adequately describe the long-wavelength $\pi_{0}^{\prime}$ data at all $\mu$ implies, of course, that spectral invariance has failed and that another photoreceptor, in addition to the rods, is influencing sensitivity.

The fitted templates and polynomials are shown in Figs 4, 6, 9 and 11 . The field points on the $\pi_{0}^{\prime}$ branches are indicated by arrows.

A less elaborate method was used to determine the cone field sensitivities. The field illuminance that raised the cone threshold by criterion amount was estimated at each $\mu$ by fitting a cubic spline to the thresholds near to the criterion elevation and interpolating to find the field point.

\section{RESULTS}

\section{Flicker threshold vs intensity curves}

An efficient way to test whether cones regulate rod flicker sensitivity is to measure rod flicker TVI profiles on a series of backgrounds of different wavelength (see e.g. Stiles, 1978). If the cones play no role, then the shape of the TVI profile should be invariant with background wavelength when the background intensities are equated for the rods; but, if cones are involved, the profile will change in shape and/or position according to the relative excitation of the cones by the background and the nature of the rod-cone interaction. In this paper, the TVI profiles are plotted in scot td. This measure of retinal illuminance takes into account the rod spectral sensitivity, $V_{\lambda}^{\prime}$, so that fields equal in scot td are rod equated.

Figure 1 shows $8 \mathrm{~Hz}$ [Fig. 1(A)] and $14 \mathrm{~Hz}$ [Fig. 1(B)] flicker TVI profiles measured in a typical, complete achromat observer $\mathrm{KN}$, who has no cone vision, against
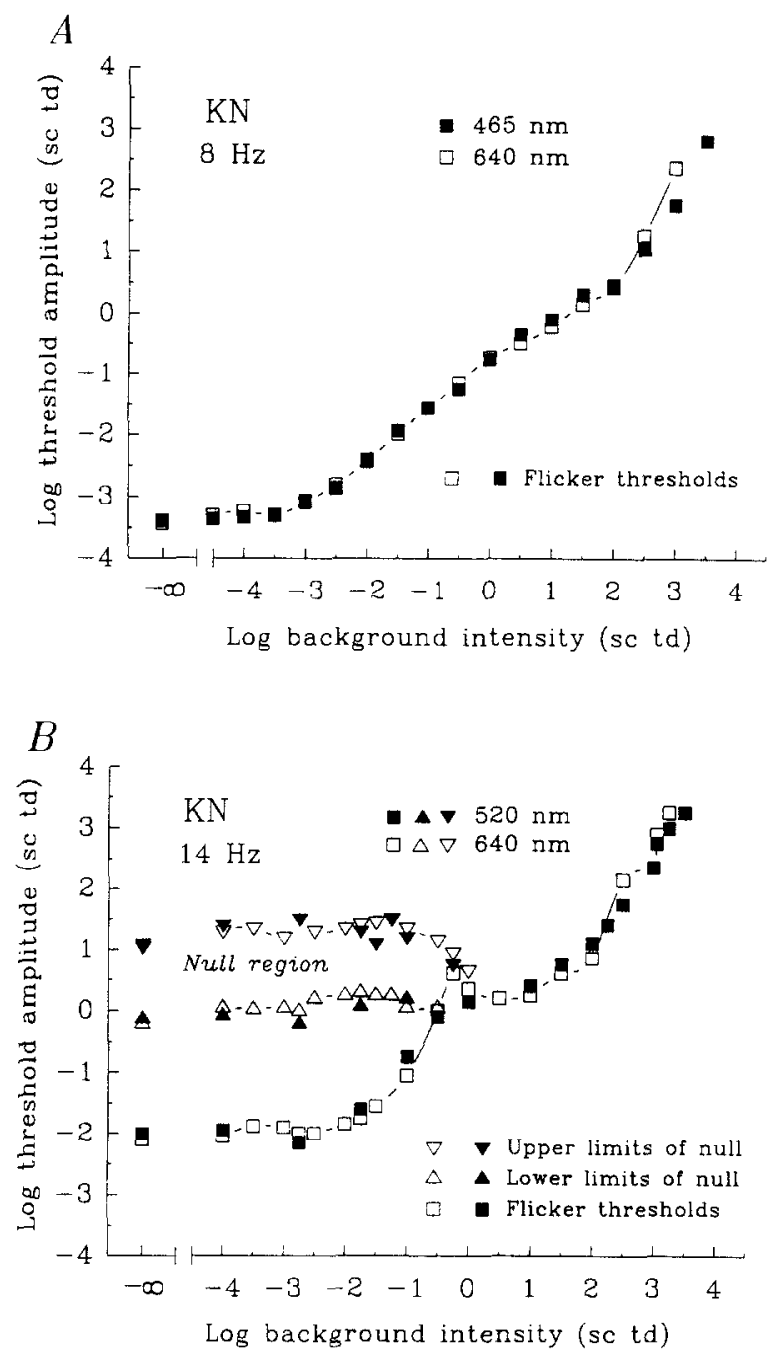

FIGURE 1. (A) $8 \mathrm{~Hz}$ and (B) $14 \mathrm{~Hz}$ detectability data for typical, complete achromat observer $\mathrm{KN}$, who has no cone vision. The $500 \mathrm{~nm}$, $6 \mathrm{deg}$ square wave flickering target was centered $13 \mathrm{deg}$ temporally from the fovea and superimposed upon a steady $16 \mathrm{deg}$ in diameter background. Superimposed data are shown for 465 or $520 \mathrm{~nm}$ (solid symbols) and $640 \mathrm{~nm}$ (open symbols) backgrounds. The squares represent the conventional flicker TVI functions; the upright and inverted triangles delimit, respectively, for the same background conditions, the nulled region at $14 \mathrm{~Hz}$ within which flicker cannot be seen.

either a 465 [Fig. 1(A) solid symbols], 520 [Fig. 1(B) solid symbols] or $640 \mathrm{~nm}$ (open symbols) background field. The squares in both panels represent the lowest amplitude at which flicker can just be seen measured as a function of intensity; the upright and inverted triangles (in the $14 \mathrm{~Hz}$ data only), the lower and upper limits, respectively, of the null region within which suprathreshold flicker is no longer seen.

At $14 \mathrm{~Hz}$, a null is found because the signals from $\pi_{0}$ and $\pi_{0}^{\prime}$ are approximately out of phase (Sharpe et al., 1989; Stockman et al., 1991). At $8 \mathrm{~Hz}$, no null is present because the signals are only approx. $90 \mathrm{deg}$ out of phase. Thus, the flicker TVI profile is not complicated by cancellation between $\pi_{0}$ and $\pi_{0}^{\prime}$. However, even though the $\pi_{0}$ and $\pi_{0}^{\prime}$ signals are in quadrature phase, there is a clear break in the $8 \mathrm{~Hz}$ TVI curve for both the achromat (near a background intensity of 0 scot td) and the normal observer (Conner, 1982; Sharpe et al., 1989, and see Figs 
6,15 and 16). This break may result from a mutually suppressive interaction between the $\pi_{0}$ and $\pi_{0}^{\prime}$ flicker signals, which reduces the overall flicker sensitivity when the two signals are both present (see also Sharpe $e t$ al., 1989 , p. 1556).

For the backgrounds shown in Fig. 1, the TVI profiles superimpose almost exactly. As would be expected in an eye that lacks functioning cone vision, there is no evidence of any cone influence on rod flicker sensitivity.

Figures 2 and 3 indicate that this is not the case for normal trichromat observers CF and RT. In both figures, $15 \mathrm{~Hz}$ flicker TVI profiles are shown for four adapting field wavelengths: $450 \mathrm{~nm}$ (A), $520 \mathrm{~nm}$ (B), $560 \mathrm{~nm}(\mathrm{C})$ and $640 \mathrm{~nm}(\mathrm{D})$. The three short-wavelength background data (solid symbols) are displayed superimposed on the $640 \mathrm{~nm}$ background data (open symbols).

The $640 \mathrm{~nm}$ field was chosen for comparison because the sensitivity difference between the peripheral cones and the rods is least at long wavelengths (see Hecht \& Hsia, 1945; Wald, 1945). Thus, for scotopically equated fields, those of long wavelength will be most effective at stimulating the cones. For both observers, the lower branches of the flicker TVI curves, the transitions between the lower and upper branches, and the boundaries of the null region align when comparisons are made between the 450,520 or $560 \mathrm{~nm}$ data and the $640 \mathrm{~nm}$
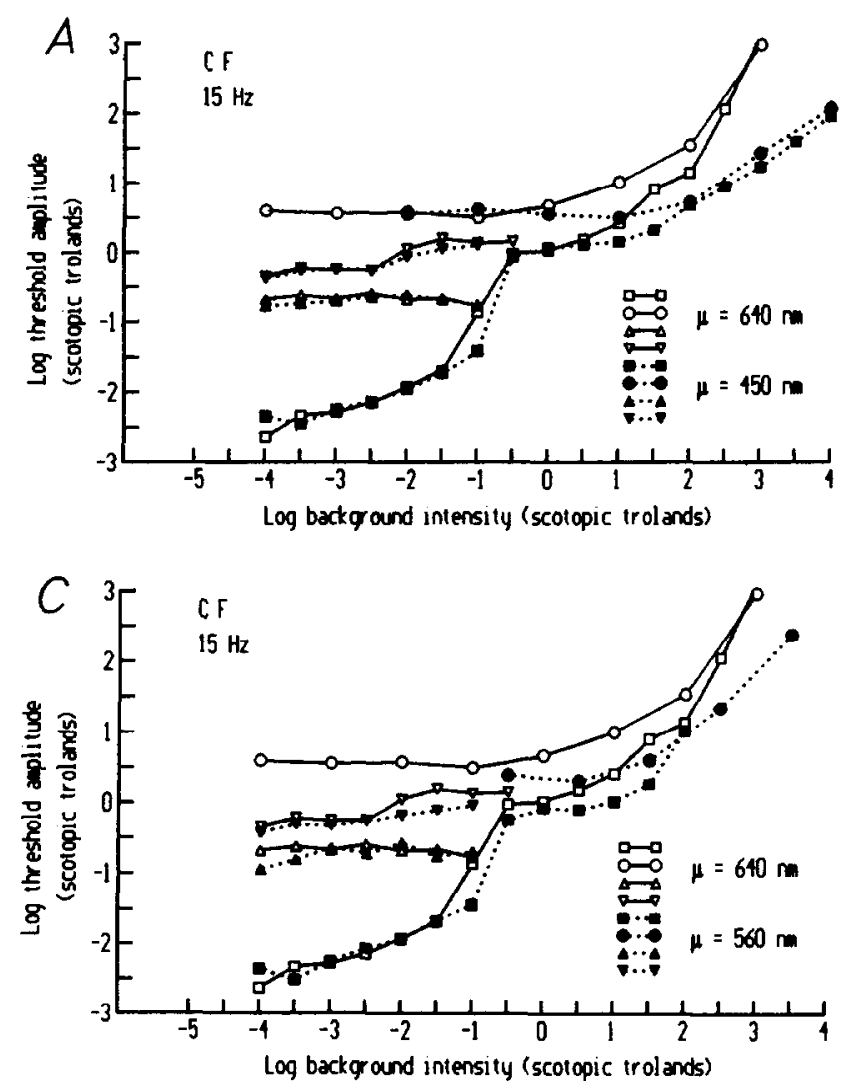

data on the rod-equated axes shown in the figures. (There is a suggestion in the data for $\mathrm{CF}$, however, that the thresholds just before the $\pi_{0} / \pi_{0}^{\prime}$ transition rise more steeply on the $640 \mathrm{~nm}$ field than on the shorter wavelength fields.)

This is not the case for the upper rod branch. Above a background intensity of about $0.25 \log$ scot td, the upper rod branch obtained on a $640 \mathrm{~nm}$ field for both subjects rises more steeply and is displaced towards lower intensities compared with the upper branches obtained on the three shorter-wavelength fields. These results suggest that cone signals arising from the steady $640 \mathrm{~nm}$ background influence rod flicker sensitivity at mesopic intensity levels, but have comparatively little effect at scotopic levels where the lower branch of the flicker TVI curve and the null region are found. It is interesting to note that the $640 \mathrm{~nm}$ upper rod branch deviates from the shorter-wavelength branches at the intensity at which the $640 \mathrm{~nm}$ background first begins to elevate the cone bleach threshold (open circles), consistent with cones elevating rod threshold.

\section{Field sensitivity functions in the normal observer}

In the normal observer, the flicker TVI curves demonstrate that sensitivity on the upper branch, but perhaps not on the lower branch, is being modified by cone
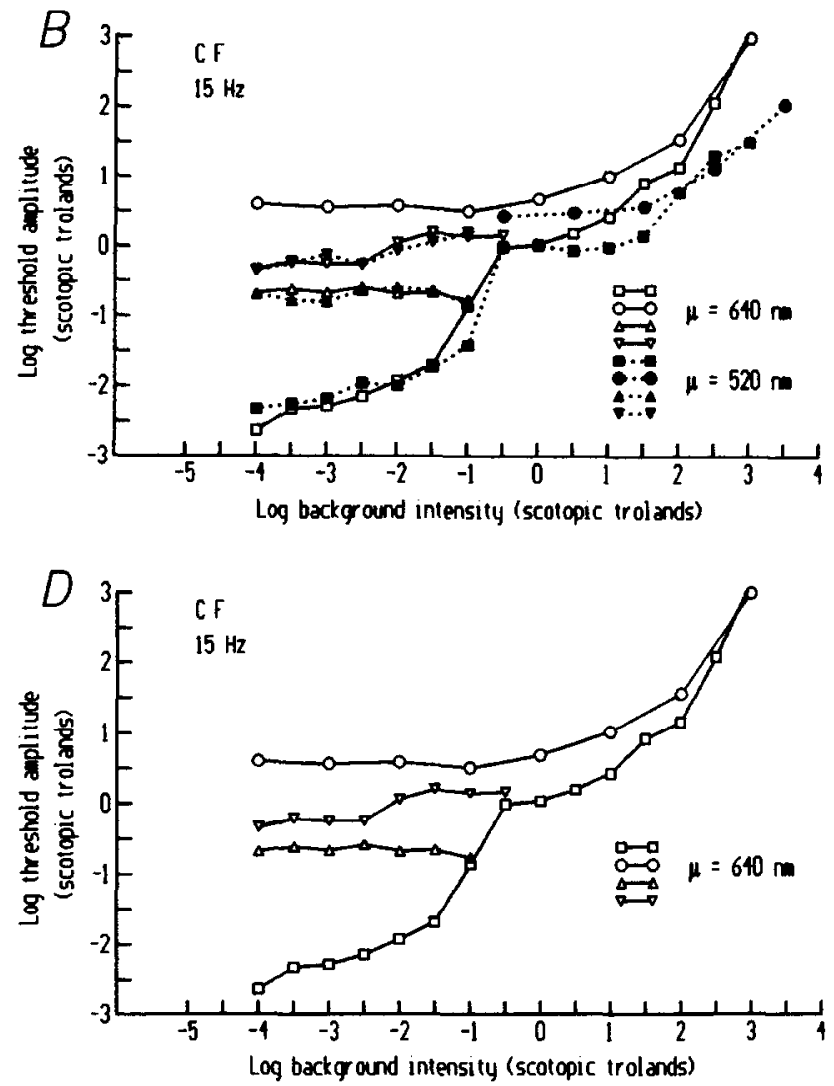

FIGURE 2. $15 \mathrm{~Hz}$ detectability data for normal trichromat observer CF. Target conditions the same as in Fig. 1, except that the entry point in the fully dilated pupil of the flickering target was displaced $3 \mathrm{~mm}$ eccentric to favor the rods. The solid squares represent the conventional flicker TVI functions measured against $450 \mathrm{~nm}$ (A), $520 \mathrm{~nm}$ (B) and $560 \mathrm{~nm}$ (C) backgrounds. The solid upright and inverted triangles delimit, respectively, for the same background conditions, the nulled region within which flicker cannot be seen. The solid circles are cone thresholds measured during the cone phase of recovery following a 7.7 log phot $t d$ sec bleach. In all four panels, the open symbols represent the same functions measured against a $640 \mathrm{~nm}$ background 

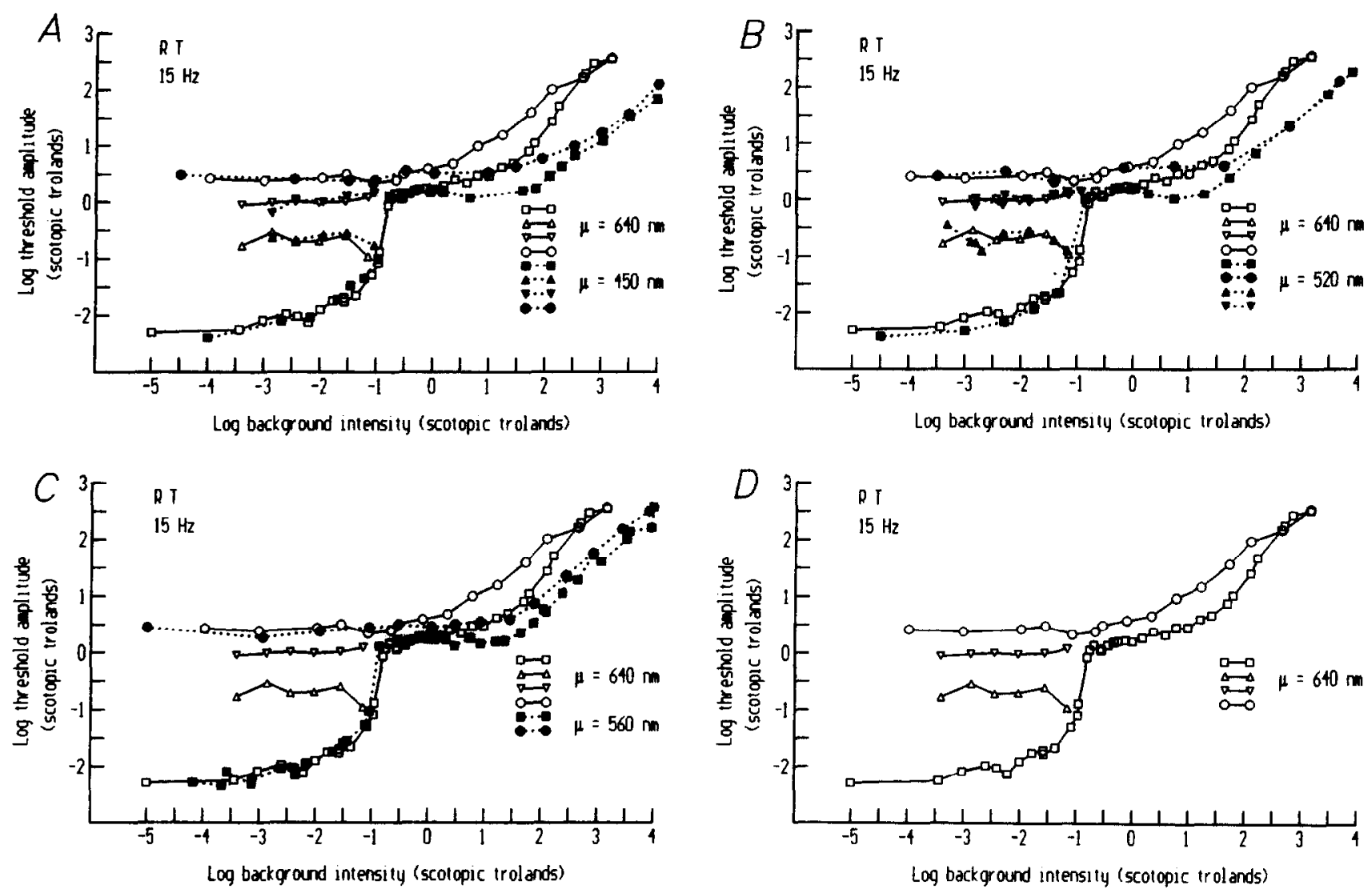

FIGURE 3. $15 \mathrm{~Hz}$ detectability data for normal trichromat observer RT. Details as for Fig. 2.

signals. To investigate the spectral properties of the two branches more precisely, we determined the field sensitivities of the lower and upper branches of 8 and $15 \mathrm{~Hz}$ flicker TVI curves. As before, the flicker curves were measured with eccentric entry $(3 \mathrm{~mm})$ of the target in the dilated pupil to favor rod detection.

$15 \mathrm{~Hz}$ data. To determine the field sensitivities of the upper and lower branches of flicker sensitivity curves in the normal observer, $\mathrm{CF}$, we measured a series of $15 \mathrm{~Hz}$ rod flicker TVI functions for 13 different background wavelengths from 450 to $640 \mathrm{~nm}$. The individual curves are shown in Fig. 4. Cone thresholds measured during the cone plateau following a bleach were also measured on each background field. For reasons of clarity, we cannot show both the rod and cone thresholds, but we have indicated rod thresholds with solid symbols, and thresholds that are likely to be determined by rods and cones or by cones alone with open symbols. In general, rod flicker thresholds fall below cone thresholds until an adapting intensity of $2.0 \mathrm{log}$ scot td at short and middle field wavelengths, and until an adapting intensity of $2.5 \mathrm{log}$ scot $\mathrm{td}$ at long wavelengths.

The transition between the two rod flicker branches is roughly independent of wavelength, always occurring near $-0.5 \log$ scot $t d$. However, the slope of the upper branch of the curve clearly steepens with background wavelength.

For the lower, $\pi_{0}$ branch, field sensitivities were derived by fitting a standard template shape to the data points, whereas for the upper, $\pi_{0}^{\prime}$ branch, they were derived by fitting a standard template (at $\mu<602 \mathrm{~nm}$ ) or by fitting second-order polynomials (at $\mu \geqslant 602 \mathrm{~nm}$; see Methods). The fitted templates and polynomials are shown in Fig. 4.

The $\pi_{0}$ field sensitivities are shown in Fig. 5 as solid circles. They correspond to the horizontal positions (converted from scot td to quantal units) of the fitted template shapes shown in Fig. 4. The $\pi_{0}^{\prime}$ field sensitivities, which are shown as open squares, correspond to the field illuminances (denoted by the vertical arrows in Fig. 4) at which the flicker threshold reached $0.25 \mathrm{log}$ scot td. For clarity, the $\pi_{0}^{\prime}$ field sensitivities are shown twice, separated by a constant vertical shift. The appropriate field illuminances were estimated by using the fitted templates or second-order polynomials, which are both shown in Fig. 4. The $\pi_{0}^{\prime}$ criterion level was chosen to be $0.25 \log$ scot $t d$, which lies $\sim 0.5 \log$ scot td above the upper branch asymptote and $\sim 0.25 \log$ unit below the cone plateau (lower intensity) asymptote $(0.5 \log \operatorname{scot}$ td). The cone field sensitivities, shown as solid triangles, correspond to the field illuminances at which the cone threshold (measured during the cone plateau following a bleach) reached $2.0 \mathrm{log}$ scot $t d$. This level is $1.50 \mathrm{log}$ units above the cone plateau asymptote.

Initially, each set of field sensitivity data was leastsquares fit by the following model of receptor interaction:

$$
\log \Pi_{\lambda}=\log \left(a L_{i}+b M_{\lambda}+c V_{\lambda}^{\prime}\right)
$$

where $\Pi_{\lambda}$ is the experimentally derived field sensitivity; $L_{i}$ and $M_{\lambda}$ are, respectively, the quantized L- and $\mathrm{M}$ cone functions of Smith and Pokorny (1975), corrected 


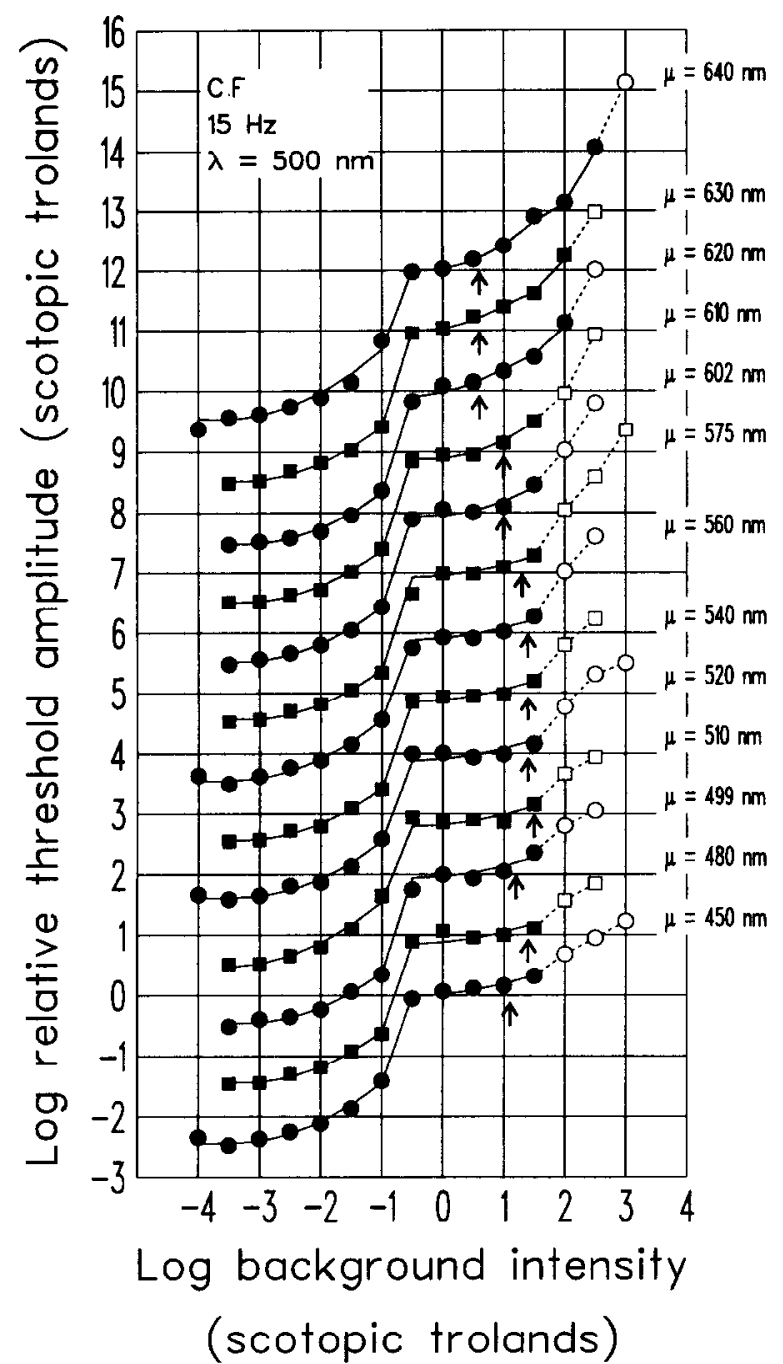

FIGURE 4. $15 \mathrm{~Hz}$ detectability data for normal trichromat observer CF. Same measurement conditions as in Fig. 2, except data are shown for 13 background wavelengths $\mu$. The curve for $\mu=450 \mathrm{~nm}$ is shown in the correct position on the threshold amplitude axis (ordinate), the other curves have been shifted by $1 \log$ unit upwards. The change from solid to open symbols indicate where the cones supersede the rods in detecting the flicker. The derivation of the functions fitted to the data points (continuous lines) is discussed in the text. The arrows indicate the field intensities at which the $\pi_{0}^{\prime}$ criterion threshold level was reached. For clarity the cone plateau thresholds and nulled region are not shown.

for macular pigment absorption (to make them more representative of the peripheral retina) according to the values tabulated in Wyszecki and Stiles (1982, p. 112); $V_{\lambda}^{\prime}$ is the quantized CIE scotopic luminosity function (Wyszecki \& Stiles, 1982, p. 789); and $a, b$ and $c$ are scaling constants. In the fits, $a$ and $b$ are constrained to be positive. (The S-cones, which might be expected to have had some influence on rod sensitivity on shortwavelength backgrounds, seem to have little or no effect.)

The relative weights (normalized so that $c=1$ ) applied to the photoreceptor spectral sensitivities $\left(L_{\lambda}: M_{\lambda}: V_{\lambda}^{\prime}\right)$ for the $\pi_{0}$ field sensitivities are $0.000: 0.000: 1.000$. The root-mean-square (r.m.s.) log error for the fit is 0.125 . Thus, the field sensitivity of the lower branch is well described by the $V_{\lambda}^{\prime}$ function alone, as shown in Fig. 5 (dotted curve).
The relative weights applied to the photoreceptor spectral sensitivities for the $\pi_{0}^{\prime}$ field sensitivity are $0.036: 0.000: 1.000$ (i.e. a linear combination of $L_{\lambda}$ and $V_{\lambda}^{\prime}$ alone; see dashed curve); and the r.m.s. log error is 0.123 . However, if the fit is repeated with $a$ constrained to be 0 , the relative weights become 0.000:0.225:1.000 (i.e. a linear combination of $M_{\lambda}$ and $V_{\lambda}^{\prime}$ alone; see continuous curve); and the r.m.s. log error deteriorates to only 0.155 . For comparison, $V_{\lambda}^{\prime}$ is also shown aligned at short wavelengths with the lower $\pi_{0}^{\prime}$ curve (dotted curve). Thus, although the $\pi_{0}^{\prime}$ field sensitivities demonstrate a clear deviation from $V_{\lambda}^{\prime}$, it is ambiguous as to whether the deviation is caused by the $\mathbf{M}$-cones and/or the L-cones. Figure 8 shows the differences between the fitted functions and the field sensitivities in greater detail.

The relative weights $\left(L_{\lambda}: M_{\lambda}\right)$ applied to the cone criterion level are $0.052: 1.000$ (the $V_{\lambda}^{\prime}$ function was assigned a weight of 0 because the rods were bleached during the measurements); and the r.m.s. log error is 0.166 . The finding of a mainly M-cone field sensitivity after a rod bleach is consistent with the $500 \mathrm{~nm}$ flickering target being detected primarily by the M-cones.

$8 \mathrm{~Hz}$ normal data. A difficulty complicating the interpretation of the $15 \mathrm{~Hz}$ field sensitivity data is that the fast rod mechanism $\left(\pi_{0}^{\prime}\right)$ and the cones are $\sim 180 \mathrm{deg}$ out of phase. Hence, the shape of the field sensitivities

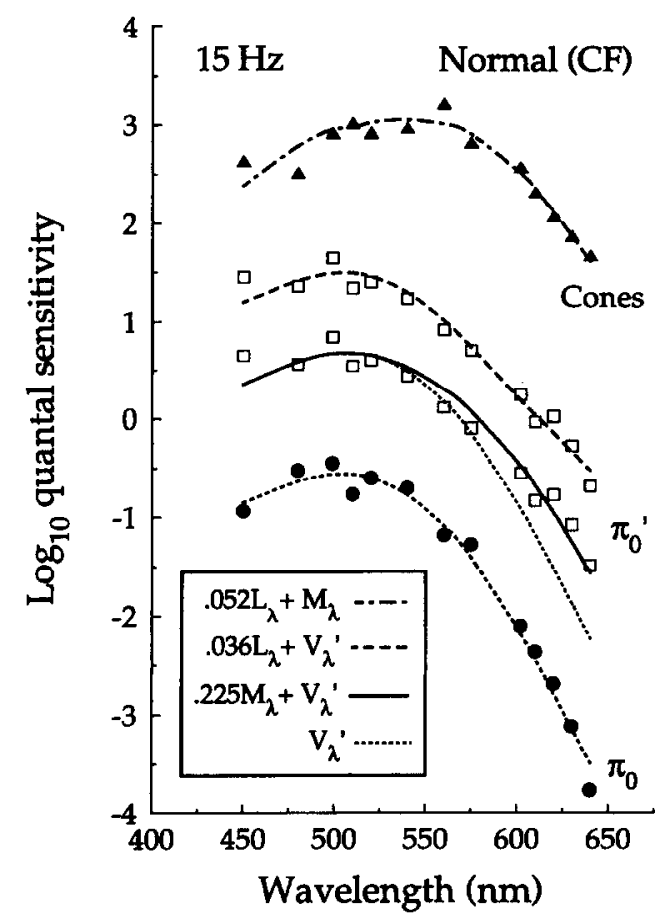

FIGURE 5. Field spectral sensitivities derived from CF's $15 \mathrm{~Hz}$ flicker thresholds given in Fig. 4. Sensitivities are shown for three criterion levels, depending on whether the target is detected by $\pi_{0}$ (solid circles), $\pi_{0}^{\prime}$ (open squares) or the cones (solid triangles). The $\pi_{0}^{\prime}$ field sensitivities are shown twice for clarity. The dotted lines represent the standard quantized CIE scotopic $\left(V_{\lambda}^{\prime}\right)$ luminosity function. The solid line drawn through the $\pi_{0}^{\prime}$ field sensitivities is the best fitting linear combination of $V_{\lambda}^{\prime}$ and the Smith and Pokorny (1975) $M_{\lambda}$ function, while the dashed line is the best fitting linear combination of $V_{\lambda}^{\prime}$ and the Smith and Pokorny (1975) $L_{\lambda}$ function. Finally, the dot-dashed line drawn through the cone field sensitivities is the best fitting linear combination of the $M_{\lambda}$ and $L_{\lambda}$ functions. The normalized weights are given in the figure. 


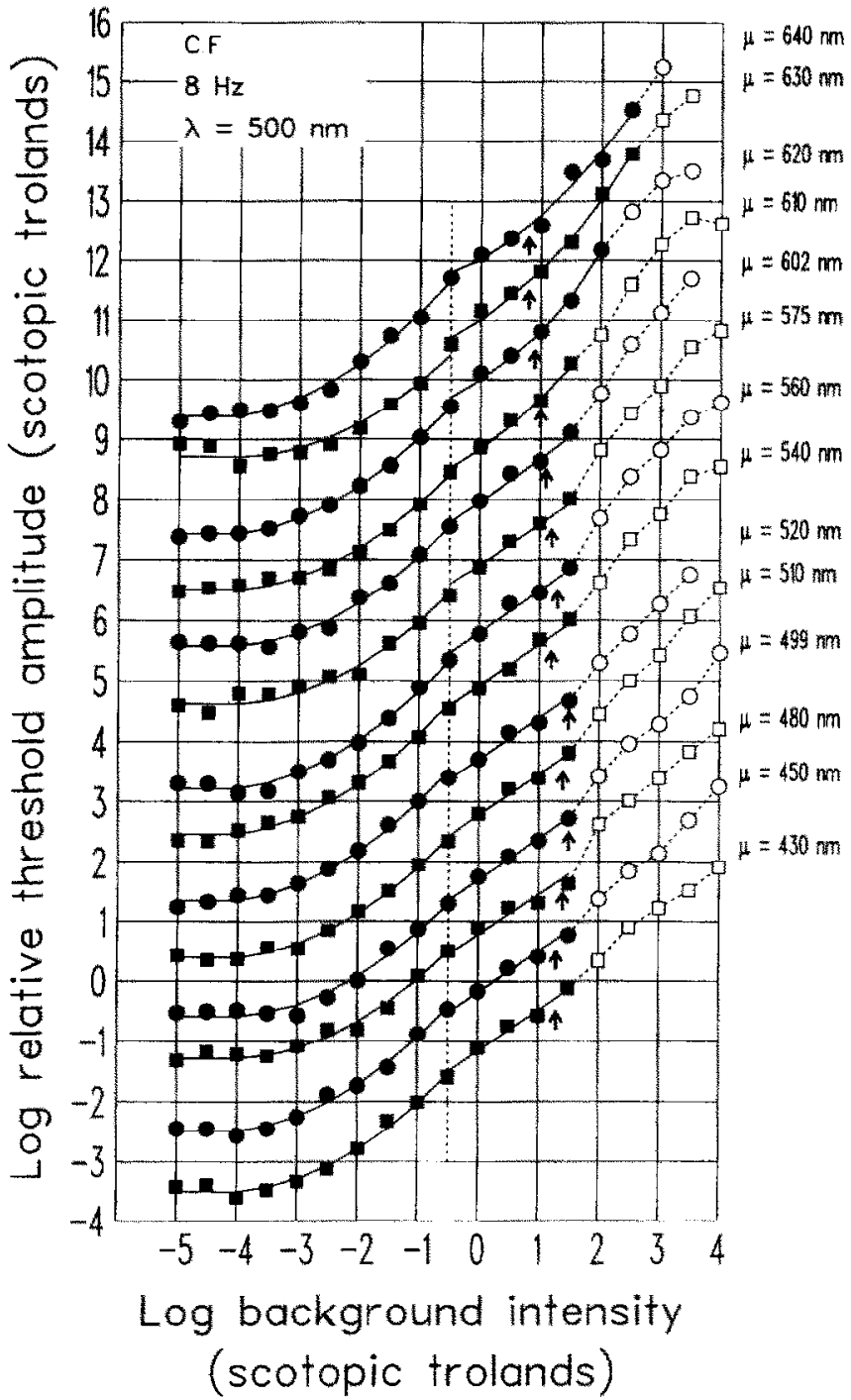

FIGURE $6.8 \mathrm{~Hz}$ detectability data for normal trichromat observer $\mathrm{CF}$. The curve for $\mu=430 \mathrm{~nm}$ is shown in the correct position on the threshold amplitude axis (ordinate), the other 13 curves have been shifted by $1 \log$ unit upwards. The vertical dashed line indicates the transition between the two branches. Other details as for Fig. 4

derived for the upper branch may be altered by destructive interference between the two processes. To obviate this problem, flicker threshold curves (Fig. 6) and field spectral sensitivity functions (Fig. 7) were measured for the same observer (CF) with an $8 \mathrm{~Hz}$ flickering light. At this frequency, the two rod pathways are only $\sim 90 \mathrm{deg}$ out of phase, so that destructive interference should not complicate the interpretation of the field sensitivities. Another advantage of using $8 \mathrm{~Hz}$ is that the clearance between the upper branch and the cone thresholds is greater. As before, the flicker curves were measured with eccentric entry $(3 \mathrm{~mm})$ of the target in the dilated pupil to favor rod detection.

The $8 \mathrm{~Hz}$ flicker curves (Fig. 6) can be divided into two branches (see vertical broken line), with the break occurring between 0.0 and $1.0 \mathrm{log}$ scot td (the change from solid to open symbols indicates where the cones supersede the rods in target detection). Field spectral sensitivities were derived for both the lower and upper branches using the methods described above.
The field sensitivities of the lower branch shown in Fig. 7 , identified as $\pi_{0}$, were derived from the horizontal positions of the template shapes shown in Fig. 6. As for the $15 \mathrm{~Hz}$ data, the $\pi_{0}$ field sensitivities are reasonably well described by the $V_{\lambda}^{\prime}$ function alone, the relative weights $\left(L_{\lambda}: M_{\lambda}: V_{\lambda}^{\prime}\right)$ being $0.000: 0.000: 1.000$. The r.m.s. $\log$ error for the fit is 0.158 .

The field sensitivities of the upper branch, identified as $\pi_{0}^{\prime}$, are for a criterion threshold of $0.0 \log$ scot td. This is $\sim 0.75 \mathrm{log}$ units above the break in the curve and $\sim 0.6 \log$ units below the cone plateau absolute threshold (which is $0.6 \mathrm{log}$ scot $t d$ ). The relative weights of the linear combination of the receptor spectral sensitivities [equation (1)] are $0.009: 0.088: 1.000$. The r.m.s. log error is 0.080 . Again, the standard errors of the parameters $a$ and $b$ are large. If the fit is repeated with $a$ constrained to be 0 , the relative weights become $0.020: 0.000: 1.000$ and the r.m.s. $\log$ error 0.092 , and if $b$ is constrained to be 0 , the relative weights become $0.000: 0.145: 1.000$ and the r.m.s. log error 0.084. Thus, the source of the cone contribution to the $\pi_{0}^{\prime}$ field sensitivity is uncertain. As in Fig. 5, the upper $\pi_{0}^{\prime}$ curve in Fig. 7 is shown fitted by the linear combination of $L_{i}$ and $V_{i}^{\prime}$ (dashed curve), and the lower $\pi_{0}^{\prime}$ curve by the linear combination of $M_{\dot{\lambda}}$ and $V_{\dot{\lambda}}^{\prime}$ (continuous curve).

In short, both the 15 and $8 \mathrm{~Hz} \pi_{0}$ field sensitivities in the normal are well described by the scotopic spectral sensitivity function, $V_{i}^{\prime}$. The $\pi_{0}^{\prime}$ field sensitivities, on the other hand, are described by a linear combination of $V_{\lambda}^{\prime}$ and $M_{\lambda}$ and/or $L_{i}$. Thus, which of the cone types is involved is hard to determine from these measurements. This is made clearer in Fig. 8 where the $\pi_{0}^{\prime}$ data (open

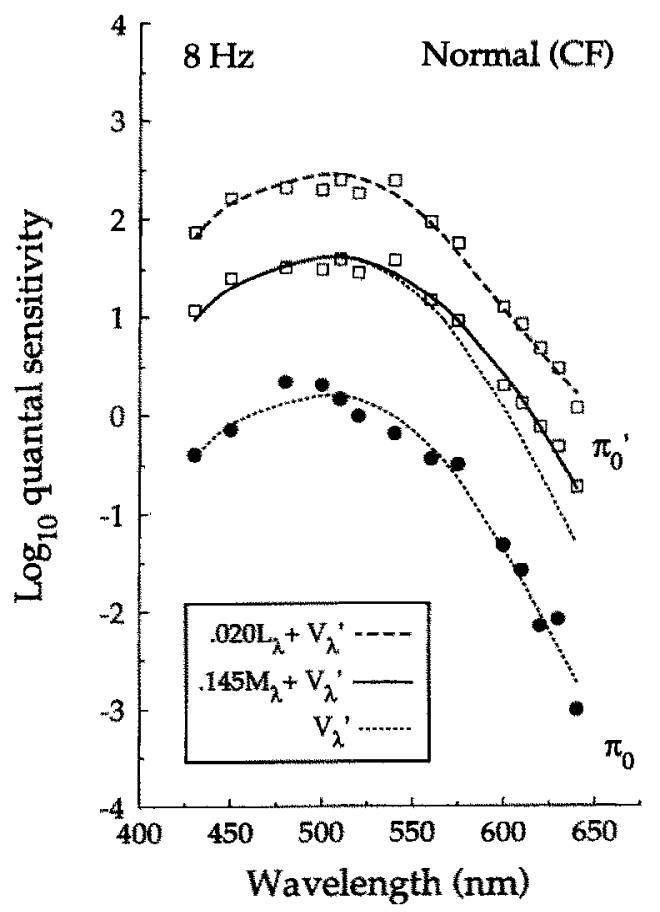

FIGURE 7. Field spectral sensitivities derived from CF's $8 \mathrm{~Hz}$ flicker thresholds given in Fig. 6. Sensitivities are shown for two criterion levels, depending on whether the target is detected by $\pi_{0}$ (solid circles) or by $\pi_{0}^{\prime}$ (open squares). Again, the $\pi_{0}^{\prime}$ field sensitivities are shown twice. Other details as for Fig. 5. 


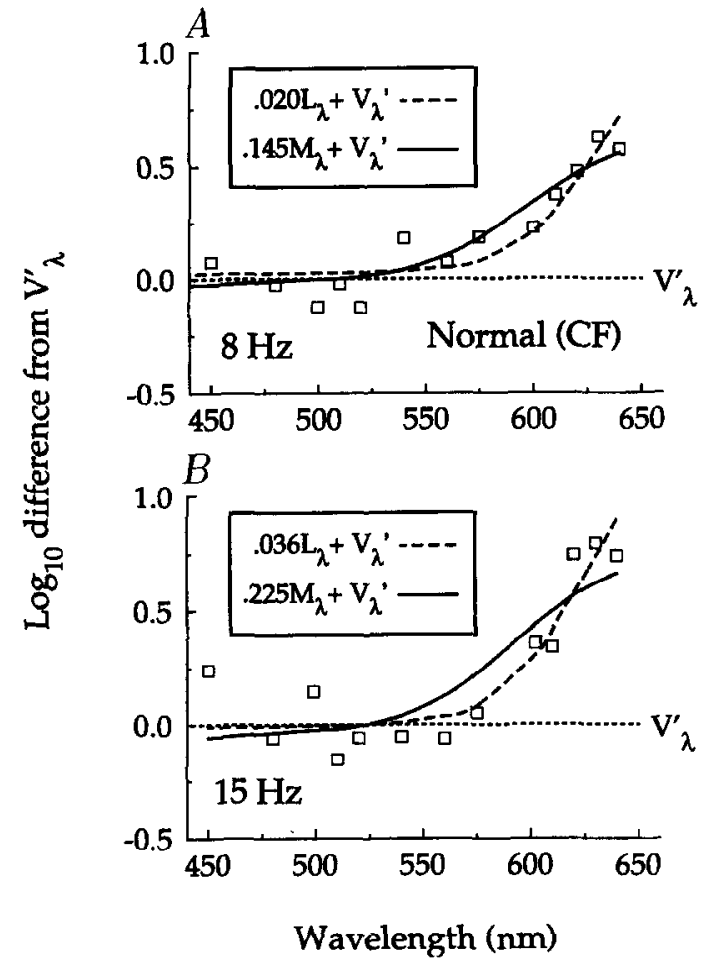

FIGURE 8. (A) $8 \mathrm{~Hz}$ and (B) $15 \mathrm{~Hz} \pi_{0}^{\prime}$ field sensitivies (open squares) from Figs 5 and 7 plotted as logarithmic differences from $V_{\lambda}^{\prime}$. Also shown are the best fitting linear combinations of $V_{\lambda}^{\prime}$ and $M_{\lambda}$ (solid line), and the best fitting linear combination of $V_{\dot{\lambda}}^{\prime}$ and $L_{\hat{\lambda}}$ (dashed line). The horizontal dotted line in each panel represents $V_{\lambda}^{\prime}$.

squares) and the fitted field sensitivity functions from Figs 5 and 7 (dashed and continuous curves) are plotted as logarithmic differences from $V_{\lambda}^{\prime}\left(V_{\lambda}^{\prime}\right.$ is represented by the horizontal dotted line). The $8 \mathrm{~Hz}$ curves are shown in [Fig. 8(A)] and the $15 \mathrm{~Hz}$ curves in [Fig. 8(B)]. The $15 \mathrm{~Hz}$ data are more consistent with the cone contribution to the field sensitivity being from the L-cones, but the $8 \mathrm{~Hz}$ data are inconclusive.

Thus, field sensitivities in the normal are not a decisive way of determining the nature of the cone influence on rod flicker sensitivity. To discover more, we must turn to dichromats.

\section{Field sensitivity functions in the dichromat observer}

$11 \mathrm{~Hz}$ flicker threshold curves and field spectral sensitivity functions were measured in a protanope (JS) and a deuteranope (NE). A flicker frequency intermediate between those used for the normal was chosen. Better isolation of the two branches is possible at $11 \mathrm{~Hz}$ than at $15 \mathrm{~Hz}$ with less complications due to destructive interference.

The $11 \mathrm{~Hz}$ flicker TVI curves for both the protanope (Fig. 9) and the deuteranope (Fig. 11) can be divided into two branches, with the break occurring between -1.0 and $0.0 \mathrm{log}$ scot td. For clarity the cone bleach thresholds are not shown: again, we indicate rod thresholds with solid symbols, and thresholds that are likely to be determined by rods and cones or by cones with open symbols.

Figure 10 shows the field spectral sensitivities for the protanope, JS, derived for $\pi_{0}$ and $\pi_{0}^{\prime}$ as well as those derived from cone plateau thresholds (not shown in Fig. 9). The field sensitivities of the lower branch, identified as $\pi_{0}$, are derived from the position of the template shapes fitted to the lower branches shown in Fig. 9. The relative weights $\left(M_{\lambda}: V_{\lambda}^{\prime}\right)$ to best fit the $\pi_{0}$ field sensitivities are 0.000:1.000; and the r.m.s. $\log$ error for the fit is 0.100 [the $L_{\lambda}$ weight in equation (1) is assumed to be 0 for this subject]. The field sensitivities of the upper branch, identified as $\pi_{0}^{\prime}$, are for a criterion flicker threshold of $0.25 \mathrm{log}$ scot td, which corresponds to $\sim 0.45 \log$ unit above the plateau of the upper branch. The arrows in Fig. 9 indicate the background intensities at which $\pi_{0}^{\prime}$ criterion threshold was reached. The relative weights to best fit the $\pi_{0}^{\prime}$ field sensitivity are $0.064: 1.000$ $\left(M_{\lambda}: V_{\lambda}^{\prime}\right)$ and the r.m.s. $\log$ error is 0.113 .

The cone field sensitivities for JS obtained during the cone plateau following a rod bleach are shown in Fig. 10 (diamonds) compared with the Smith and Pokorny M-cone (dot-dashed curve). The cone field sensitivities are consistent with JS having only an M-cone longer wavelength pigment.

Figure 12 shows the field spectral sensitivities for the deuteranope, NE, derived from the lower and upper rod

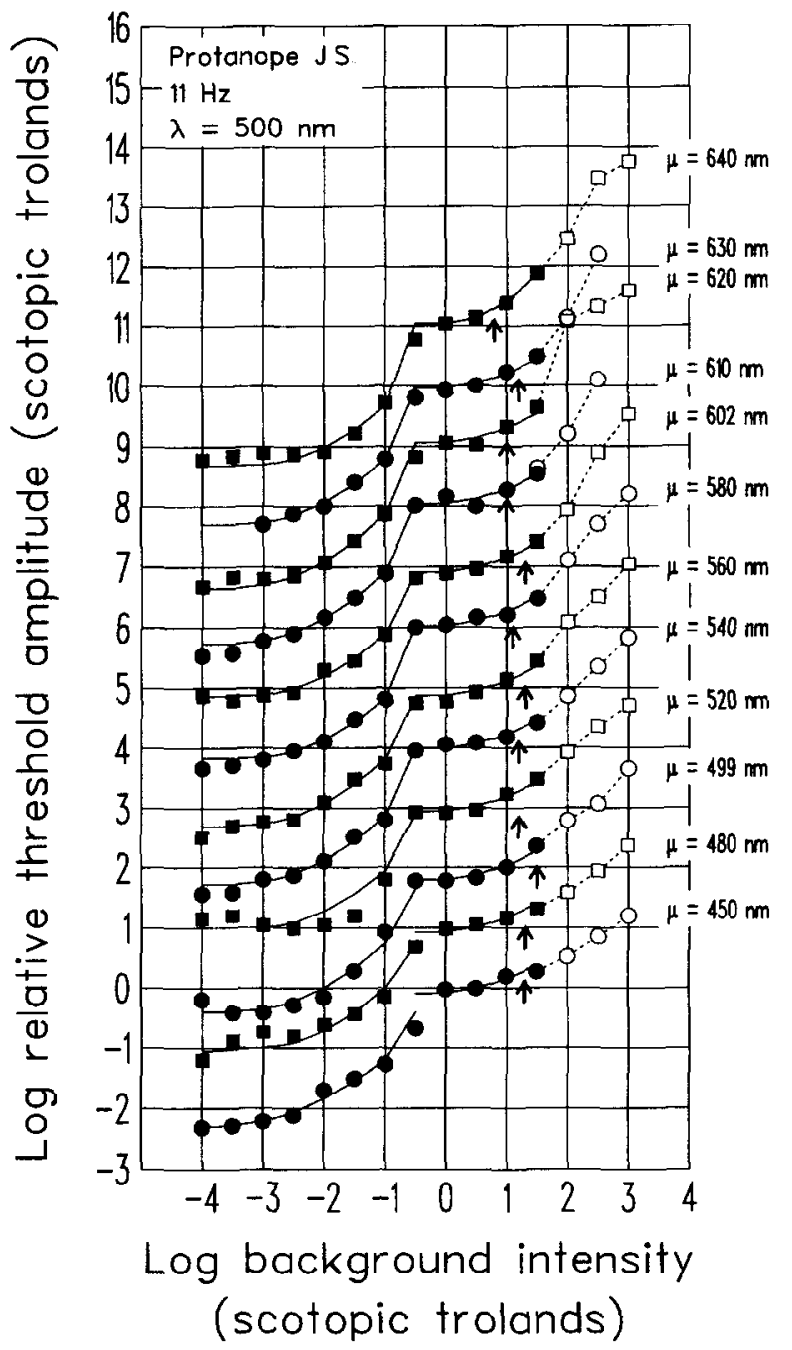

FIGURE 9. $11 \mathrm{~Hz}$ detectability data for protanope observer JS. The curve for $\mu=450 \mathrm{~nm}$ is shown in the correct position on the threshold amplitude axis (ordinate), the other 11 curves have been shifted by $1 \mathrm{log}$ unit upwards. Other details as for Fig. 4. 


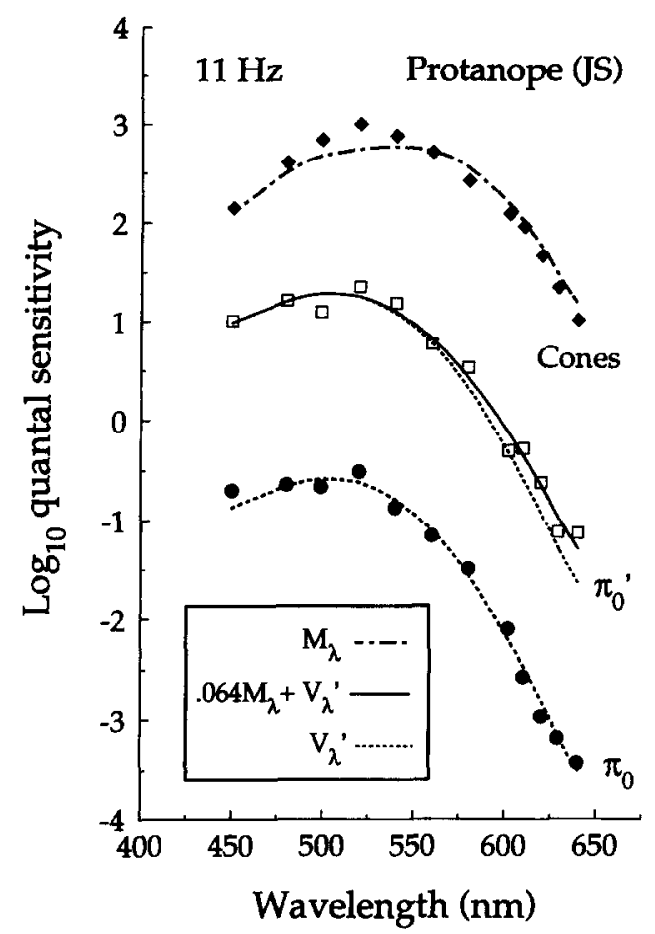

FIGURE 10. Field spectral sensitivities derived from protanope JS's $11 \mathrm{~Hz}$ flicker thresholds given in Fig. 9. For the protanope, the $\pi_{0}^{\prime}$ field sensitivities (open squares) are shown only once. The solid line drawn through them is the best fitting linear combination of $V_{\lambda}^{\prime}$ and $M_{\lambda}$. The dot-dashed line drawn through the cone field sensitivities (solid diamonds) is $M_{i}$. Other details as for Fig. 5.

branches shown in Fig. 11 and from cone bleach thresholds (not shown in Fig. 11). The field sensitivities of the lower branch correspond to the template positions shown in Fig. 11. The relative weights $\left(L_{i}: V_{\lambda}^{\prime}\right)$ to fit the $\pi_{0}$ field sensitivities are $0.000: 1.000$; and the r.m.s. $\log$ error for the fit is 0.107 [the $M_{\lambda}$ weight in equation (1) is assumed to be 0 for this subject]. Thus, for all of our subjects, the normal, the protanope and the deuteranope, the $\pi_{0}$ field spectral sensitivity measured to $640 \mathrm{~nm}$ is well described by $V_{\lambda}^{\prime}$.

The field sensitivities of the upper branch are for a flicker threshold of $0.00 \mathrm{log}$ scot td, which is $\sim 0.50 \log$ unit above the plateau of the upper branch. The relative weights $\left(L_{\lambda}: V_{\lambda}^{\prime}\right)$ for the $\pi_{0}^{\prime}$ criterion level are $0.013: 1.000$; and the r.m.s. log error is 0.138 . The cone field sensitivities obtained following a rod bleach (diamonds) shown in Fig. 12 compared with the Smith and Pokorny L-cone function (dot-dashed curve) are consistent with NE having only an L-cone longer wavelength pigment.

Though the field sensitivities obtained in the normal observer were inconclusive, those obtained in the dichromats reveal an L-cone influence on $\pi_{0}^{\prime}$ in the deuteranope and an M-cone influence on $\pi_{0}^{\prime}$ in the protanope. This suggests that both cone types can influence $\pi_{0}^{\prime}$.

\section{Flicker thresholds following background exchange}

The results presented so far indicate that both the Land the $\mathrm{M}$-cones can influence the sensitivity regulation of the upper, $\pi_{0}^{\prime}$ branch of the 8,11 and $15 \mathrm{~Hz}$ ficker TVI functions. Yet, there seems to be little cone influence on the lower, $\pi_{0}$ branch. This result may arise because $\pi_{0}$ predominates only at low, scotopic adaptation levels at which the cones are relatively unadapted (cf. rod and cone flicker thresholds in Figs 2 and 3). Or it could suggest that the sensitivity regulation of $\pi_{0}$, unlike $\pi_{0}^{\prime}$, proceeds independently of the cones. To try to resolve this issue, we remeasured the $15 \mathrm{~Hz}$ TVI curves on an adapting field that abruptly changed wavelength at a rate of $0.5,1$ or $2 \mathrm{~Hz}$. The change was effected by alternating a $679 \mathrm{~nm}$ background and a $480 \mathrm{~nm}$ background, the luminances of which were scotopically equated at all luminance levels. This alternation was invisible to the rods, but produced a large cone transient (the difference in cone excitation for these two components, calculated according to $V_{\lambda}$, the photopic luminous efficiency function, is approx. $3.8 \mathrm{log}$ units). Since transient adaptation is generally more effective than steady state adaptation in raising threshold (e.g. Craik, 1938; Crawford, 1947), these conditions should reveal if there is any cone influence at adaptation levels low enough to affect the $\pi_{0}$ branch. On the other hand, if the sensitivity regulation of $\pi_{0}$ is entirely independent of the

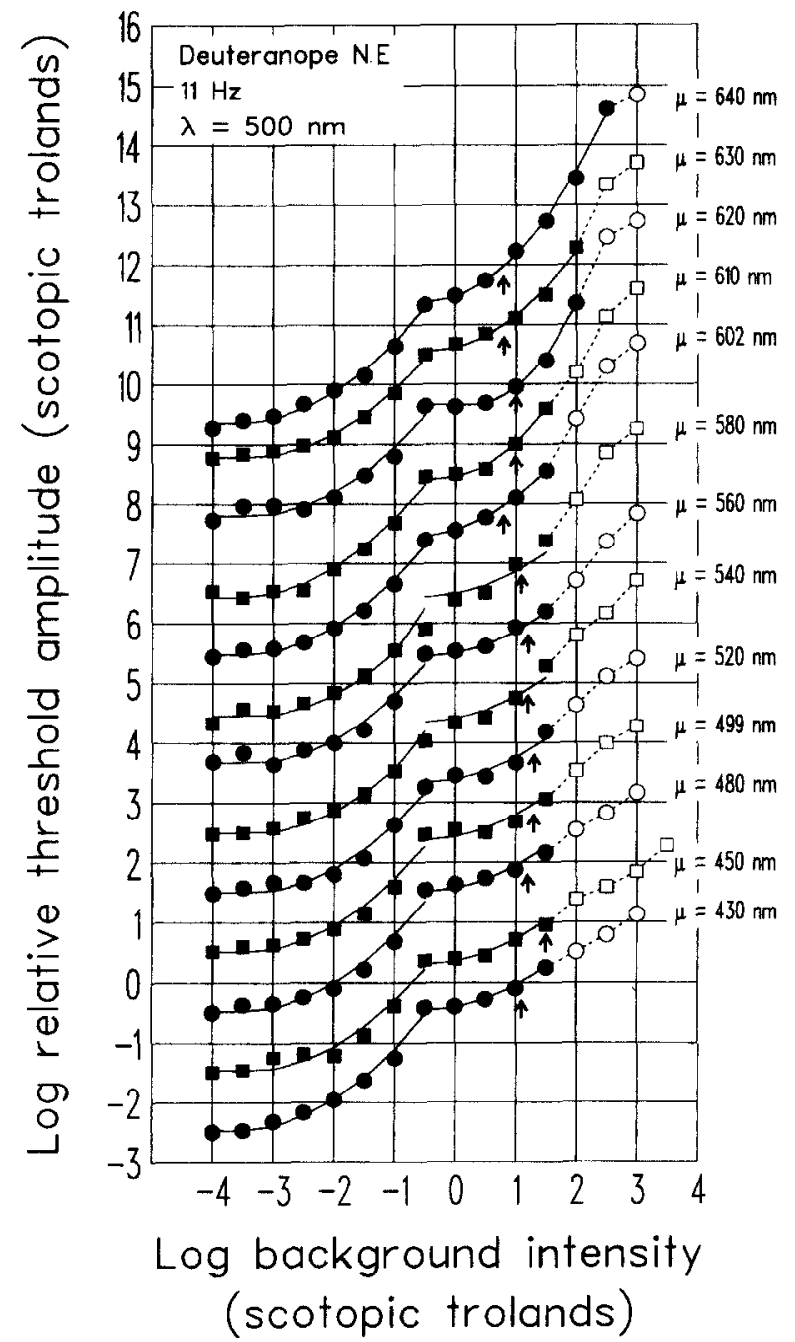

FIGURE $11.11 \mathrm{~Hz}$ detectability data for deuteranope observer NE. Same measurement conditions as in Fig. 9. The curve for $\mu=430 \mathrm{~nm}$ is shown in the correct position on the threshold amplitude axis (ordinate), the other 12 curves have been shifted by $1 \log$ unit upwards. Other details as for Fig. 4. 


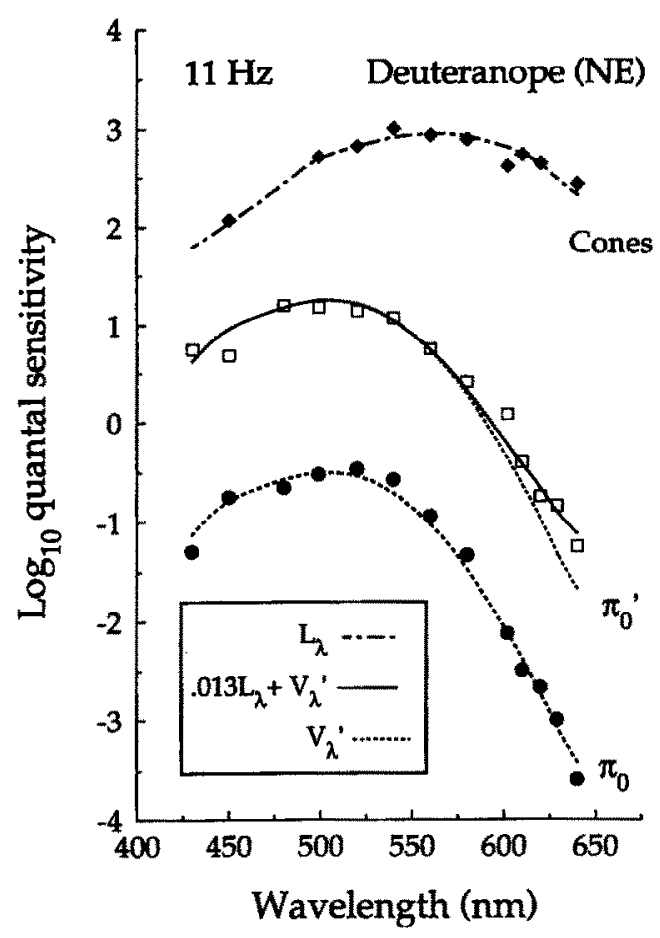

FIGURE 12. Field spectral sensitivities derived from deuteranope NE's $11 \mathrm{~Hz}$ flicker thresholds given in Fig. 11. For the deuteranope, the $\pi_{0}^{\prime}$ field sensitivities (open squares) are shown only once. The solid line drawn through them is the best fitting linear combination of $V_{\lambda}^{\prime}$ and $L_{\lambda}$. The dot-dashed line drawn through the cone field sensitivities (solid diamonds) is $L_{i}$. Other details as for Fig. 5.

cones, the $\pi_{0}$ branch obtained on the alternating backgrounds should not differ from that found on a steady background.

Figures 13 and 14 show the results for normal observers $\mathrm{CF}$ and $\mathrm{JH}$ for field substitution rates of $0.5(\mathrm{~A})$, 1.0 (B) or 2.0 (C) Hz. Four types of threshold were measured: the threshold for detecting the target's flicker (open squares); the lower and upper limits of the nulled region within which flicker cannot be seen (upright and inverted open triangles); and cone thresholds measured during the cone phase of recovery following a $7.7 \mathrm{log}$ photopic bleach (open circles). At background alternations of 0.5 and $1 \mathrm{~Hz}$, the subject set the threshold on the red, $679 \mathrm{~nm}$ adapting field, ignoring the flicker seen on the $480 \mathrm{~nm}$ field. At $2 \mathrm{~Hz}$, the threshold was set without regard to the color of the background, since the exchange was too rapid for the subject to be able to set threshold on only the red background. For comparison, the same rod thresholds are shown obtained on a steady adapting field of $480 \mathrm{~nm}$, which does not strongly excite the cones at these levels. It can be seen that at all three alternation frequencies, the rod flicker thresholds on the lower and upper branches are elevated compared with those measured on the steady $480 \mathrm{~nm}$ adapting field. The steepening of the lower branch coincides with the desensitization of the cones, as indicated by the parallel steepening of the cone plateau threshold curve. Notice also that at all three frequencies the null region broadens slightly, but that its position is relatively unaffected. This suggests that the cones reduce the sensitivity of $\pi_{0}$ and $\pi_{0}^{\prime}$ about equally.
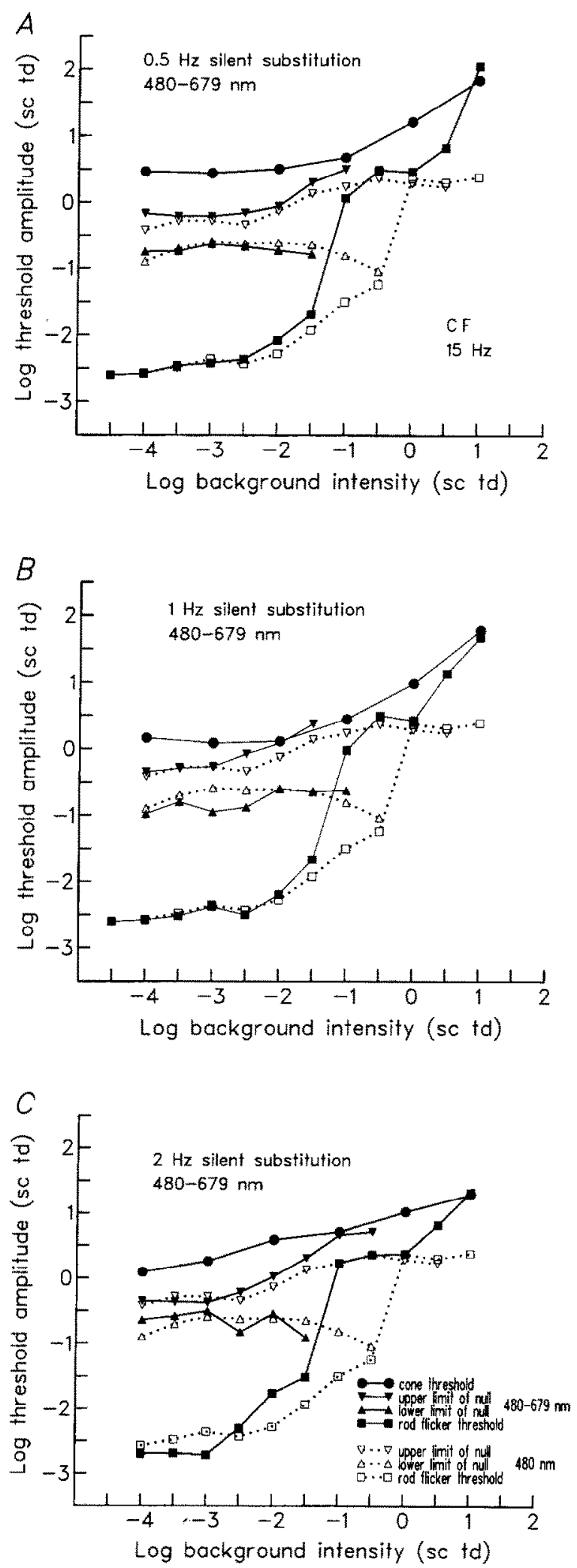

FIGURE 13. $15 \mathrm{~Hz}$ detectability data for normal trichromat observer $\mathrm{CF}$, measured under silent substitution conditions. Rod-equated backgrounds of 480 and $679 \mathrm{~nm}$ were exchanged at the rate of 0.5 (A), 1 (B) and 2 (C) Hz. Solid symbols represent thresholds measured on the $679 \mathrm{~nm}$ background following the exchange; open symbols, thresholds measured on a steady $480 \mathrm{~nm}$ background (control). Target conditions the same as in Fig. 2. The squares represent the conventional rod flicker threshold. The upright and inverted triangles delimit, respectively, the nulled region within which flicker cannot be seen. The circles are cone thresholds measured during the cone phase of recovery following a $7.7 \mathrm{log}$ phot td sec bleach. 

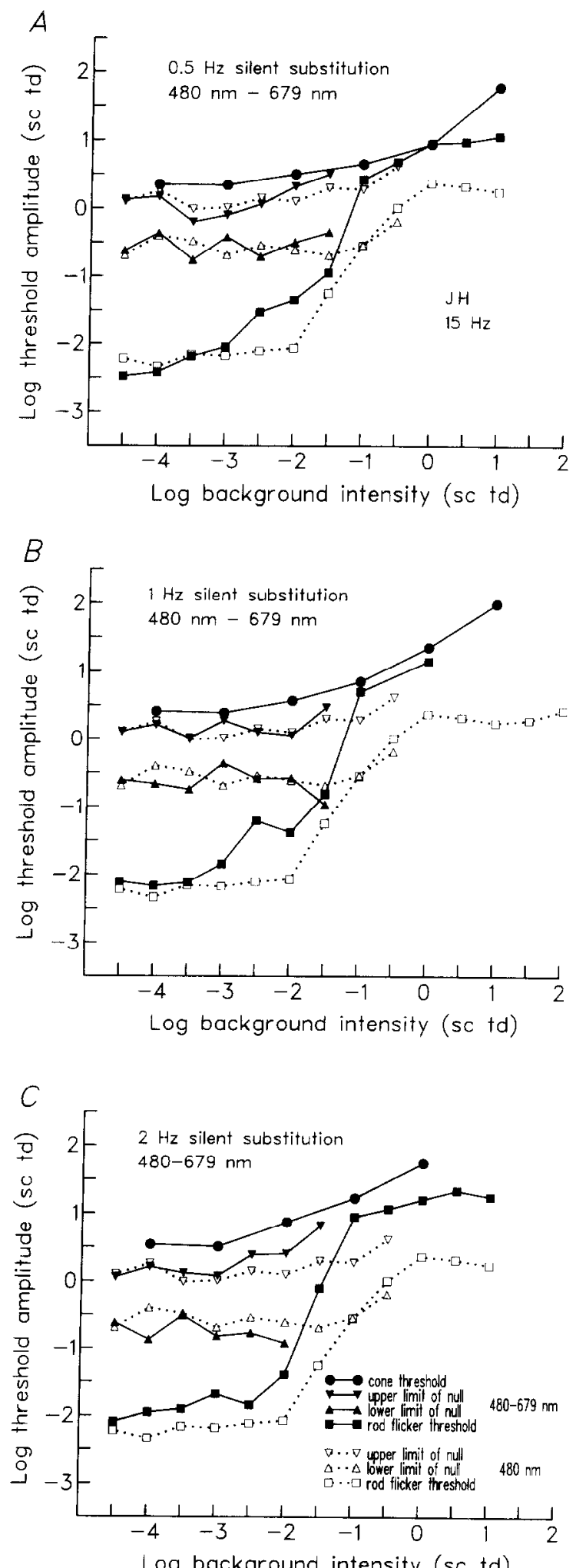

FIGURE 14. $15 \mathrm{~Hz}$ detectability data for normal trichromat observer JH measured under silent substitution conditions. Details as for Fig. 13.

A problem with the interpretation of the results of this experiment, however, is that the loss of sensitivity caused by transient cone signals may occur at a comparatively central site in the visual pathway, after the signals from $\pi_{0}$ and $\pi_{0}^{\prime}$ recombine (see Discussion). This site may be distinct from the sensitivity regulating site(s) at which steady cone signals raise rod threshold. In the next section, however, we present evidence that cone signals produced by steady, long-wavelength fields can, in fact, elevate $\pi_{0}$ threshold.

\section{Steady fields of long wavelength}

In the steady-field experiments described above, we measured rod flicker thresholds and field sensitivities on steady backgrounds from 430 to $640 \mathrm{~nm}$. And, we found scant evidence that the cones influence the lower or $\pi_{0}$ branch. In light of the results of the last experiment, in which an elevation of $\pi_{0}$ threshold was found following a background exchange from 480 to $679 \mathrm{~nm}$, we decided to extend our steady-field flicker sensitivity measurements to include backgrounds as long as $680 \mathrm{~nm}$. If there is a small cone influence on $\pi_{0}$, we might expect it to be most apparent on a deep-red background. To ensure that we did not miss any small threshold elevations in the neighborhood of the $\pi_{0}$ and $\pi_{0}^{\prime}$ transition, we made measurements at smaller background steps of $0.25 \mathrm{log}$ unit (rather than the $0.5 \mathrm{log}$ unit step used previously).

Figure 15(A) shows $8 \mathrm{~Hz}$ flicker TVI profiles measured in a normal observer, LTS. The measurement conditions were the same as in previous experiments, save sinusoidal instead of square-wave flicker was used. The individual profiles are vertically separated for clarity. We derived an average template shape to describe the lower, $\pi_{0}$ branch at $\mu=500$ and $575 \mathrm{~nm}$; it is shown in Fig. 15(A) fitted to the data points on the lower, 500 and $575 \mathrm{~nm}$ TVI branches (continuous lines). At $\mu=640$, 650 and $680 \mathrm{~nm}$, the same template shape is shown aligned with the data points obtained at the lower background intensities. At higher intensities, as the transition from $\pi_{0}$ to $\pi_{0}^{\prime}$ approaches, the template shape is distinctly shallower than the TVI data. Since the deviations from the template first occur at or before $-1 \log \operatorname{scot} t d$, well before the $\pi_{0}$ and $\pi_{0}^{\prime}$ transition, they suggest an influence of the cones on $\pi_{0}$.

The curves fitted to the upper, $\pi_{0}^{\prime}$ branches in Fig. 15(A) are second-order polynomials individually fitted at each $\mu$. The slope of the data clearly steepens with increasing background wavelength.

The differences between the shapes of both the lower and upper branches of the flicker TVI curves, as a function of $\mu$, can also be seen clearly in Fig. 15(B), in which the data are shown superimposed. Though not as large as the marked differences between the $\pi_{0}^{\prime}$ branches, the smaller differences between the $\pi_{0}$ branches are still apparent. Comparable results for normal observer CF are shown in Fig. 16.

Figure 17(A) shows $15 \mathrm{~Hz}$ flicker TVI profiles for LTS. Again, a template shape was derived to describe the lower, $\pi_{0}$ branch at $\mu=500$ and $575 \mathrm{~nm}$. It is shown fitted to the data points on the lower, TVI branches at all $\mu$ (continuous lines). As in Fig. 15(A), the curves fitted to the upper, $\pi_{0}^{\prime}$ branches in Fig. 17(A) are second-order polynomials individually fitted at each $\mu$. At $15 \mathrm{~Hz}$, the data points on the lower branches do not deviate substantially from the template shape until just before the $\pi_{0}$ to $\pi_{0}^{\prime}$ transition. These deviations, which occur at 

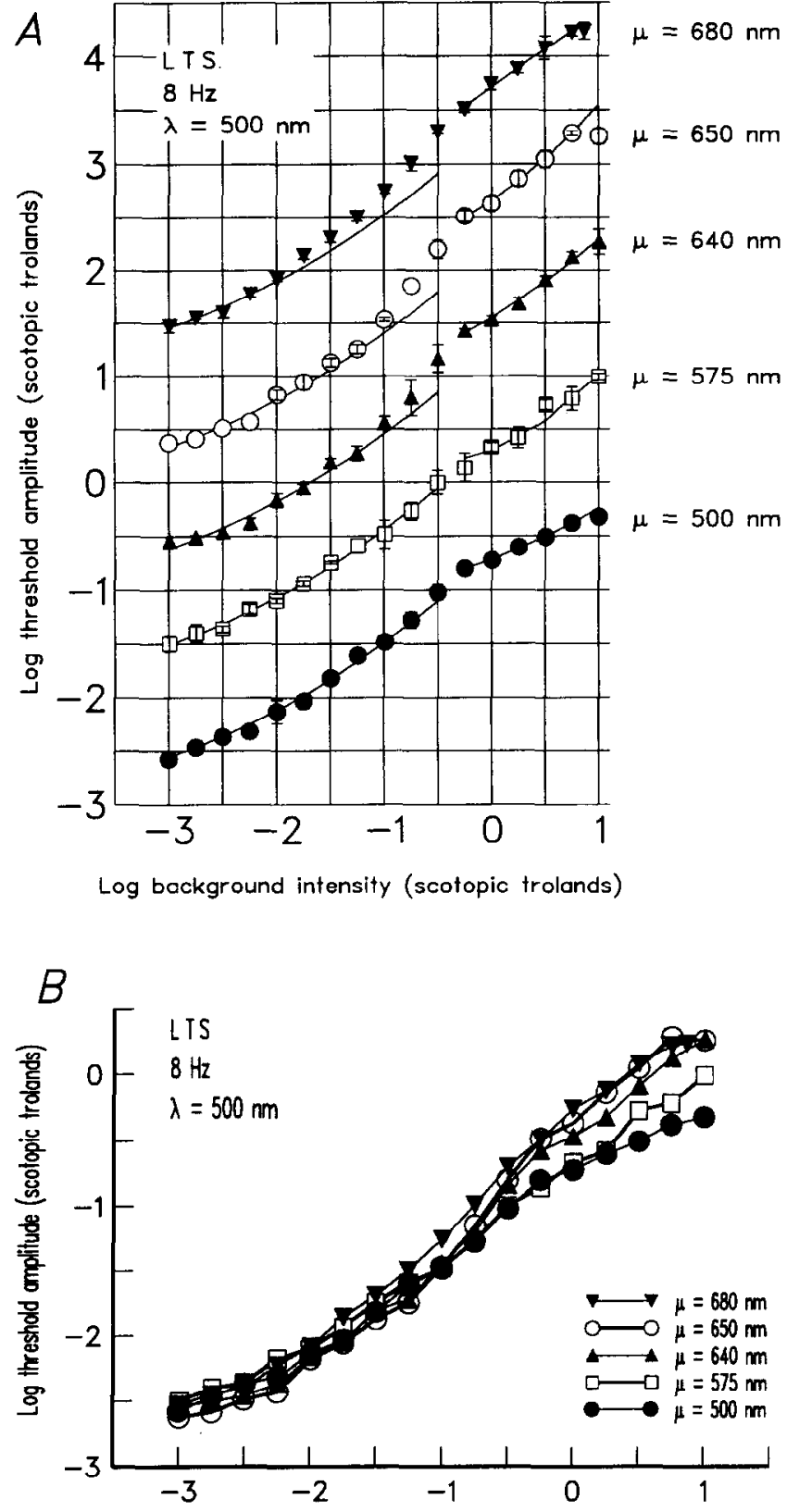

Log bockground intensity (scotopic trolands)

FIGURE $15.8 \mathrm{~Hz}$ flicker detectability data for normal trichromat observer LTS for $\mu=500$ (solid circles), 575 (open squares), 640 (solid triangles), 650 (open circles) and 680 (inverted, solid triangles) $\mathrm{nm}$. (A) The data for each $\mu$ are shown vertically separated by $1 \log$ unit steps (the ordinate is correct for $\mu=500 \mathrm{~nm}$ ). The function (solid line) drawn through each lower, TVI branch is the second-order polynomial that best fits the averaged lower branch data at $\mu=500$ and $575 \mathrm{~nm}$. The function fitted to each upper, TVI branch is the best fitting second-order polynomial individually fitted at each $\mu$. See text for details. The error bars indicate one standard deviation of the mean. (B) The data for $\mu=500,575,640,650$ and $680 \mathrm{~nm}$ are shown superimposed.

background intensities between -0.5 and $0.0 \log$ scot td, can be seen more clearly in Fig. 17(B). A similar deviation in the $15 \mathrm{~Hz}$ data for CF can also be seen in Fig. 2. Although these deviations are consistent with a cone influence on $\pi_{0}$, the situation is more complicated at 15 than at $8 \mathrm{~Hz}$, since the $\pi_{0}$ and $\pi_{0}^{\prime}$ signals are out of phase. As a result, changes in sensitivity before the $\pi_{0}$ and $\pi_{0}^{\prime}$ transition could be caused by changes in either the $\pi_{0}^{\prime}$ signal or the $\pi_{0}$ signal.

One interesting feature of Figs 15(B), 16 and 17(B) is that the vertical position of the lower asymptote of the $\pi_{0}^{\prime}$ branch depends strongly on $\mu$. This provides further evidence of a cone influence on $\pi_{0}^{\prime}$.

\section{DISCUSSION}

At scotopic background illuminances, where $\pi_{0}$ predominates, the shapes of the 8,11 and $15 \mathrm{~Hz}$ flicker TVI profiles measured under steady-state conditions on background fields ranging from 430 to $640 \mathrm{~nm}$ are approximately independent of background wavelength (see Figs 4, 6, 9 and 11). Under these conditions, then, the regulation of rod flicker sensitivity depends primarily on signals from rods. A small cone influence on $\pi_{0}$ can be demonstrated near to the $\pi_{0}$ to $\pi_{0}^{\prime}$ transition if deep-red steady background fields are used (Figs 15-17) or if large cone transients are generated by silently substituting rod-equated backgrounds (Figs 13 and 14).

At still higher illuminance levels, where $\pi_{0}^{\prime}$ predominates, the flicker TVI profiles are not independent of background wavelength: increasing the background wavelength at constant scotopic illuminance substantially elevates rod flicker threshold. Thus the regulation of rod flicker sensitivity at mesopic levels clearly depends on signals from both rods and cones.

\section{The nature of the cone influence on $\pi_{0}^{\prime}$}

Two previous studies have investigated the cone influence on rod flicker sensitivity. Conner (1982) added supplemental backgrounds of either $469,500,546,600$ or $681 \mathrm{~nm}$ to a main red background (Kodak No. 92 Wratten filter) that doubled the total scotopic illuminance. He found that the shape of both branches of the double-branched $12 \mathrm{~Hz}$ flicker profile measured on the combined backgrounds was independent of the wavelength of the supplemental background. Unfortunately, this is a very limited test of the independence of rod

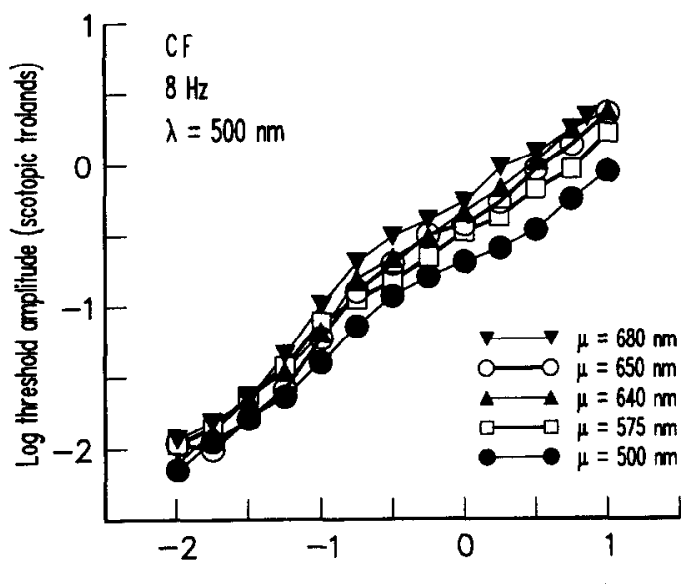

Log bockground intensity (scotopic trolends)

FIGURE 16. $8 \mathrm{~Hz}$ flicker detectability data for normal trichromat observer CF for $\mu=500$ (solid circles), 575 (open squares), 640 (solid triangles), 650 (open circles) and 680 (inverted, solid triangles) $\mathrm{nm}$. Details as for Fig. $15(\mathrm{~B})$. 


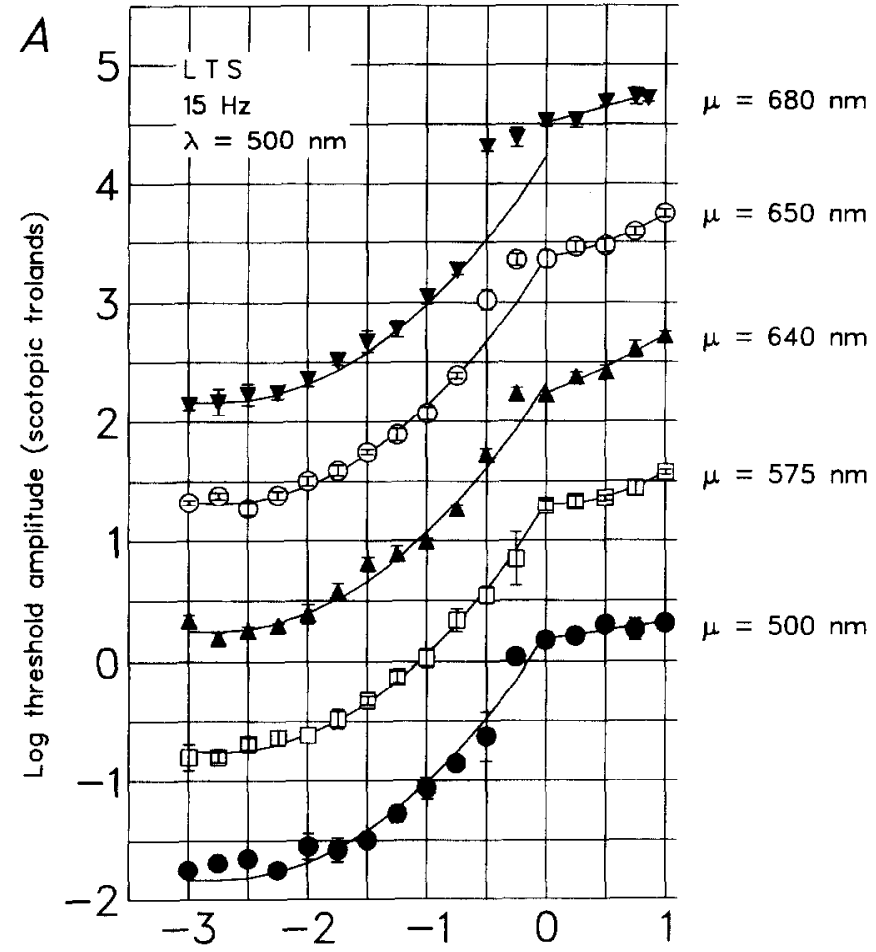

Log background intensity (scotopic trolands)

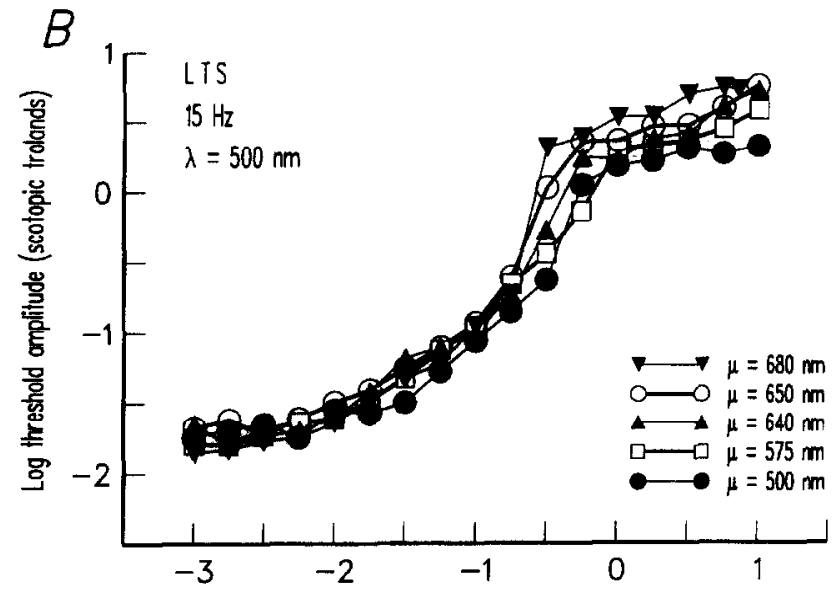

Log bockground intensity (scotopic trolands)

FIGURE 17. $15 \mathrm{~Hz}$ flicker detectability data for normal trichromat observer LTS for $\mu=500$ (solid circles), 575 (open squares), 640 (solid triangles), 650 (open circles) and 680 (inverted, solid triangles) $\mathrm{nm}$. Details as for Fig. 15.

adaptation, because although the scotopically-equated supplemental fields of $469,500,546$ and $600 \mathrm{~nm}$ double the scotopic luminance, they cause only a comparatively small increase in photopic luminance. For such fields, rod adaptive independence should approximately hold, as Conner found. Only the added $681 \mathrm{~nm}$ background causes an increase in photopic luminance that is comparable to the scotopic increase. But, even then, compared with the small overall rise in threshold caused by doubling the scotopic luminance (a rise of $<0.3 \log$ unit), the extra elevation due to increasing the cone excitation is likely to be smaller than the variability seen in the data.
More recently, Knight et al. (1990, 1991) have reported in abstract form comparable measurements to those reported here. They concluded that the sensitivity regulation on the upper, $\pi_{0}^{\prime}$ branch of the $15 \mathrm{~Hz}$ rod flicker TVI curve is mediated by the rods and the $\mathrm{M}$-cones. However, an exclusive role for the $\mathbf{M}$-cones is clearly inconsistent with the deuteranope's $\pi_{0}^{\prime}$ field sensitivities (Fig. 12), which show a marked cone influence at long wavelengths.

Rod field sensitivity measurements using flashed targets

The possibility that the desensitization of the cones by steady backgrounds can increase rod flicker threshold accords with other observations that the sensitivity of the rods to a flashed target is not determined by quantal absorptions in the rods alone but by quantal absorptions in both the rods and the cones. The dependence of the field adaptation of the rods on the cones has been shown for flashed rod targets using steady and transient adapting fields (e.g. Makous \& Boothe, 1974; Frumkes \& Temme, 1977; Ingling, Lewis, Loose \& Meyers, 1977; Makous \& Peeples, 1979; Sharpe et al., 1989, 1992). Since the dependence is only marked on long-wave adapting fields, the influence of the $\mathrm{M}$-cones (which are less sensitive to long-wave lights than the L-cones) is always likely to seem less than the influence of the L-cones, even though the two could be equally effective (in quantal terms) in elevating rod threshold.

\section{Other considerations}

(i) The relationship between the lower and upper $15 \mathrm{~Hz}$ $T V I$ branches and $\pi_{0}$ and $\pi_{0}^{\prime}$. We would like to emphasize that a simple correspondence between the two branches of the flicker TVI curves and $\pi_{0}$ and $\pi_{0}^{\prime}$ should not be assumed. According to our model, much of the doublebranched TVI profile (e.g. the steeply ascending portion of the curve and the early portion of the upper branch) reflects destructive interference between $\pi_{0}$ and $\pi_{0}^{\prime}$ (see Sharpe et al., 1989). Consequently, the relative shapes and positions of both branches depend in part upon signals from both $\pi_{0}$ and $\pi_{0}^{\prime}$, and, as a result, the " $\pi_{0}$ " and " $\pi_{0}^{\prime \prime " ~ f i e l d ~ s e n s i t i v i t i e s ~ g i v e n ~ a b o v e ~ m u s t ~ b e ~ t o ~ s o m e ~}$ extent mixed $\pi_{0}$ and $\pi_{0}^{\prime}$ functions.

(ii) Is $\pi_{0}$ exclusively a "rod" pathway? Our steady-state results suggest that at low scotopic illumination levels, the field sensitivity of $\pi_{0}$ is approximately rod-like, but that it can be influenced by cones near the $\pi_{0}$ to $\pi_{0}^{\prime}$ transition when field wavelengths longer than $640 \mathrm{~nm}$ are used. These results suggest that sensitivity regulation of $\pi_{0}$, like that of $\pi_{0}^{\prime}$, is not independent of cones. The relatively small cone influence of $\pi_{0}$ compared with $\pi_{0}^{\prime}$ may reflect the fact that $\pi_{0}$ predominates at background intensities at which the cone signals from the background are simply too small to produce a very large elevation of rod threshold. As Figs 15(B), 16 and 17(B) illustrate, we do not find an abrupt change in field sensitivity as detection passes from $\pi_{0}$ to $\pi_{0}^{\prime}$, as would be expected if the sensitivity regulation of $\pi_{0}^{\prime}$, but not $\pi_{0}$, depended on cones. Indeed, the deviations from a scotopic field sensitivity first seem to occur when the 
backgrounds begin to raise cone threshold, irrespective of whether detection is by $\pi_{0}$ or $\pi_{0}^{\prime}$.

(iii) Rod-cone flicker interactions and field sensitivity. At $15 \mathrm{~Hz}$, there is a $360 \mathrm{deg}$ phase difference between $\pi_{0}$ and the cones, and a 180 deg phase difference between $\pi_{0}^{\prime}$ and the cones (Sharpe et al., 1989). Consequently, if the $\pi_{0}^{\prime}$ field sensitivities are measured using a $15 \mathrm{~Hz}$ target that is too close to the cone flicker threshold (so that the cones also contribute to flicker detection), the resulting field sensitivity will be complex, since the cones will alter the overall flicker threshold through rod-cone flicker interactions (as well as through steady signals produced by the background). Moreover, the size of the rod-cone flicker interaction will depend on the field wavelength, because changing the field wavelength will change the relative sensitivity of the rod and cones to the flickering target. Since the target typically used to favor rod detection is $500 \mathrm{~nm}$, the most likely rod-cone flicker interaction is between the $\mathbf{M}$-cones (which are more sensitive to the $500 \mathrm{~nm}$ target than the L-cones) and the rods. However, we were careful to avoid this problem by using the Stiles-Crawford effect to improve rod isolation and by estimating the rod field sensitivity well below cone threshold. Thus, we doubt that rod-cone interactions in flicker detection strongly influenced our results.

(iv) Rod-rod ficker interactions. The relative prominence of $\pi_{0}$ and $\pi_{0}^{\prime}$ varies with retinal illuminance: $\pi_{0}$ predominates at low scotopic illuminances and is superseded by $\pi_{0}^{\prime}$ at high ones. Some evidence suggests that, relative to the $\pi_{0}^{\prime}$ signal, the $\pi_{0}$ signal actually saturates at intensities above the null (Sharpe et al., 1989). If $\pi_{0}$ does saturate, then the flicker threshold at $15 \mathrm{~Hz}$ (which is elevated because of destructive interference between signals from $\pi_{0}$ and $\pi_{0}^{\prime}$ ) will fall. Such a downturn can be seen in the TVI profiles at $14 \mathrm{~Hz}$ for KN (Fig. 1) and at $15 \mathrm{~Hz}$ for RT (Fig. 3): after the transition from the lower to upper branches the flicker threshold falls. This effect is unlikely to be caused by flicker interactions between the cones and $\pi_{0}^{\prime}$, since their signals are out of phase at this frequency.

(v) Other ficker measurements. There is a considerable literature on the effect of steady annuli or backgrounds on rod-cone interactions in flicker sensitivity (e.g. Goldber, Frumkes \& Nygaard, 1983; Alexander \& Fishman, 1984, 1985; Coletta \& Adams, 1984; Arden \& Hogg, 1985; Frumkes, Naarendorp \& Goldberg, 1986). It is unclear how these interactions are related either to the doubled-branched $15 \mathrm{~Hz}$ TVI function, which depends primarily on rod-rod flicker interactions (see Fig. 1), or to the sensitivity regulation of the upper branch.

(vi) The site of the effect of the cone transient. Conceivably, the cone transient introduced by alternating the 678 and $480 \mathrm{~nm}$ fields could have its effect on rod flicker sensitivity at one of three sites: (1) at an early site before $\pi_{0}$ and $\pi_{0}^{\prime}$ signals diverge; (2) after the signals diverge, but before they recombine to produce the flicker null; or (3) at some more central site, after they recombine. In each case, the effect on the $15 \mathrm{~Hz}$ rod flicker threshold, and on the lower and upper limits of the $15 \mathrm{~Hz}$ null would be different. In case (1), there should be no change in the overall shape of the $15 \mathrm{~Hz}$ flicker profile, but an overall loss in sensitivity. In case (3), the flicker threshold would rise and the null would broaden. Case (2) is more complicated, since the cone transient could differentially alter the sensitivities of $\pi_{0}$ and $\pi_{0}^{\prime}$. If the sensitivity of only $\pi_{0}^{\prime}$ is reduced, the flicker threshold would remain the same (or perhaps decrease slightly), and both the lower and the upper limit would increase. In contrast, if the sensitivity of only $\pi_{0}$ is reduced, the flicker threshold would rise, and both the lower limit and upper limit would decrease. Finally, if the sensitivities of $\pi_{0}$ and $\pi_{0}^{\prime}$ are reduced together by a similar amount, the flicker threshold would increase, and the null would broaden [i.e. as case (3)]. In general, the results (Figs 13 and 14) seem to be consistent either with case (3) or with case (2) with an equal reduction in the sensitivities of $\pi_{0}$ and $\pi_{0}^{\prime}$. They do not support a differential influence of cones on the sensitivity of either $\pi_{0}$ and $\pi_{0}^{\prime}$. Thus, the loss of sensitivity introduced by the cone transient could be central to the site at which the signals from $\pi_{0}$ and $\pi_{0}^{\prime}$ recombine, and central to the sites at which the sensitivities of $\pi_{0}$ and $\pi_{0}^{\prime}$ are regulated under steady state conditions.

(vii) A multiplicative model of receptor interaction. We used an additive model [equation (1)] of receptor interactions to fit our rod field sensitivities. However, an alternative model is suggested by the work of Rushton (1959) and Alpern and Campbell (1962a, b). Rushton was ablc to explain the mesopic spectral response of the frog ganglion cell, by assuming that the logarithm of the sensitivity is the weighted mean of the logarithms of the scotopic and photopic sensitivities; and Alpern and Campbell (1962b) demonstrated that human pupillomotor spectral sensitivity curves measured at mesopic intensities are better described by a weighted logarithmic addition than by a weighted linear addition. We employed a similar model, in which we assumed that the spectral sensitivity is the weighted mean of the logarithm of the $L_{\lambda}, M_{\lambda}$ and $V_{\lambda}^{\prime}$. In general, the fits were marginally worse than the additive model [equation (1)].

(viii) Physiological considerations. Elsewhere we consider in detail the anatomical substrate of the two rod pathways (see Sharpe et al., 1989; Sharpe \& Stockman, 1991). It perhaps suffices here to say that the $\pi_{0}$ pathway probably conveys rod signals from the rod spherules to the ganglion cells via the rod bipolar and amacrine AII cells (and cone bipolars). The substrate of the $\pi_{0}^{\prime}$ pathway remains uncertain. It may be routed to the cone bipolars via electrical gap junctions between the cone pedicles and the neighbouring rod spherules (see above), which would suggest that $\pi_{0}^{\prime}$ should be more susceptible than $\pi_{0}$ to the influence of cone signals. The results of the experiments in which we alternated backgrounds (Figs 13 and 14) or used long-wavelength steady backgrounds (Figs 15-17) suggest that cones can reduce the sensitivity of both $\pi_{0}$ and $\pi_{0}^{\prime}$. These results do not therefore support a model in which the sensitivity of $\pi_{0}$ is exclusively regulated by rods. Such a result would seem highly unlikely, in any case, since rod and cone signals (if both 
are present) travel in shared pathways beyond the amacrine cells, even at low light levels. The nature of the cone influence on rod sensitivity is therefore unlikely to be an unambigous way of distinguishing between possible retinal pathways for $\pi_{0}$ or $\pi_{0}^{\prime}$.

\section{REFERENCES}

Alexander, K. R. \& Fishman, G. A. (1984). Rod-cone interaction in flicker perimetry. British Journal of Ophthalmology, 68, 303-309.

Alexander, K. R. \& Fishman, G. A. (1985). Rod-cone interaction in flicker perimetry: Evidence for a distal retinal locus. Documenta Ophthalmologica, 60, 3-36.

Alexander, K. R. \& Kelly, S. A. (1984). The influence of cones on rod saturation with flashed backgrounds. Vision Research, 24, 507-511

Alexander, K. R., Kelly, S. A. \& Morris, M. A. (1986). Background size and saturation of the rod system. Vision Research, 26, 299-312.

Alpern, M. \& Campbell, F. W. (1962a). The physiology of mixed and cone spectral response curves. Journal of Physiology, London, 156, $12-13 \mathrm{P}$.

Alpern, M. \& Campbell, F. W. (1962b). The spectral sensitivity of the consensual light reflex. Journal of Physiology, London, 164, 478-507.

Arden, G. B. \& Hogg, C. R. (1985). Rod-cone interaction and analysis of retinal disease. British Journal of Ophthalmology, 69, 404-415.

Coletta, N. J. \& Adams, A. J. (1984). Rod-cone interactions in flicker detection. Vision Research, 24, 1333-1340.

Conner, J. D. (1982). The temporal properties of rod vision. Journal of Physiology, London, 332, 139-155.

Conner, J. D. \& MacLeod, D. I. A. (1977). Rod photoreceptors detect rapid flicker. Science, 195, 689-699.

Cornsweet, T. N. (1962). The staircase method in psychophysics. American Journal of Psychology, 75, 485-491.

Craik, K. J. W. (1938). The effect of adaptation on differential brightness adaptation. Journal of Physiology, London, 92, 406421

Crawford, B. H. (1947). Visual adaptation in relation to brief conditioning stimuli. Proceedings of the Royal Society of London B, 134, 283-302.

Daw, N. W., Jensen, R. J. \& Brunken, W. J. (1990). Rod pathways in mammalian retinae. Trends in Neuroscience, 13, 110-115.

Flamant, F. \& Stiles, W. S. (1948). The directional and spectral sensitivities of the retinal rods to adapting fields of different wavelengths. Journal of Physiology, London, 107, 187-222.

Frumkes, T. E. \& Temme, L. A. (1977). Rod-cone interactions in human scotopic vision-II. Cones influence rod increment thresholds. Vision Research, 17, 673-679.

Frumkes, T. E., Naarendorp, F. \& Goldberg, S. H. (1986). The influence of cone adaptation upon rod mediated flicker. Vision Research, 26, 1167-1176

Goldberg, S. H., Frumkes, T. E. \& Nygaard, R. W. (1983). Inhibitory influence of unstimulated rods in the human retina: Evidence provided by examining cone flicker. Science, 221, 180-182.

Hecht, S. \& Hsia, Y. (1945). Dark adaptation following light adaptation to red and white lights. Journal of the Optical Society of America, 35, 261-267.

Hecht, S., Shlaer, S., Smith, E. L., Haig, C. \& Peskin, J. C. (1938). The visual functions of a completely color blind person. American Journal of Psychology, 123, 94-95.

Hecht, S., Shlaer, S., Smith, E. L., Haig, C. \& Peskin, J. C. (1948). The visual functions of the complete color-blind. Journal of General Physiology, 31, 459-472.

Hess, R. F. \& Nordby, K. (1986a). Spatial and temporal limits of vision in the achromat. Journal of Physiology, London, 371, 365-385.

Hess, R. F. \& Nordby, K. (1986b). Spatial and temporal properties of human rod vision in the achromat. Journal of Physiology, London, $371,387-406$

Hess, R. F., Nordby, K. \& Pointer, J. S. (1987). Regional variation of contrast sensitivity across the retina of the achromat: Sensitivity of human rod vision. Journal of Physiology, London, 388, 101-119.

Ingling, C., Lewis, A. L., Loose, D. R. \& Myers, J. K. (1977). Cones change rod sensitivity. Vision Research, 17, 555-563.
Knight, R., Buck, S. L. \& Sanocki, E. (1991). Subthreshold flicker additivity of $\mathbf{M}$ - and L-cone signals with fast $\left(\pi_{0}^{\prime}\right)$ rod signals. Optical Society of America Technical Digest, 17, 41 .

Knight, R., Sanocki, E. \& Buck, S. L. (1990). Field adaptation of dual rod mechanisms in the detection of $15 \mathrm{~Hz}$ flicker. Investigative Ophthalmology and Visual Science (Suppl.), 31, 494.

Makous, W. \& Boothe, R. (1974). Cones block signals from rods. Vision Research, 14, 285-294.

Makous, W. \& Peeples, D. (1979). Rod-cone interaction: Reconciliation with Flamant and Stiles. Vision Research, 19, 695-698.

Nordby, K. N. \& Sharpe, L. T. (1988). The directional sensitivity in the human achromat. Journal of Physiology, London, 399, 267-281.

Nordby, K. N., Stabell, B. \& Stabell, U. (1984). Dark adaptation of the human rod system. Vision Research, 24, 841-849.

Rushton, W. A. H. (1959). Excitation pools in the frog's retina. Journal of Physiology, London, 149, 327-345.

Sharpe, L. T. \& Nordby, K. (1990). The photoreceptors in the achromat. In Hess, R., Sharpe, L. T. \& Nordby, K. (Eds), Night vision: Basic, clinical and applied aspects. Cambridge: Cambridge University Press

Sharpe, L. T. \& Stockman, A. (1991). Dual rod pathways. In Valberg, A. \& Lee, B. B. (Eds), From pigments to perception. Advances in understanding visual processes. New York: Plenum Press.

Sharpe, L. T. \& Volbrecht, V. (1990). Estimating middle- and longwave cone sensitivity with large, long and tiny, brief targets. Perception, 19, 745-757

Sharpe, L. T., Fach, C. C. \& Stockman, A. (1992). The field adaptation of the human rod visual system. Journal of Physiology, London, 445, 319-343.

Sharpe, L. T., van Norren, D. \& Nordby, K. (1988). Pigment regeneration, visual adaptation and spectral sensitivity in the achromat. Clinical Vision Sciences, 3, 9-17.

Sharpe, L. T., Stockman, A. \& MacLeod, D. I. A. (1989). Rod flicker perception: Scotopic duality, phase lags and destructive interference. Vision Research, 29, 1539-1559.

Sharpe, L. T., Fach, C. C., Nordby, K. \& Stockman, A. (1989). The incremental threshold of the rod visual system and Weber's law. Science, 244, 354-356.

Smith, V. C. \& Pokorny, J. (1975). Spectral sensitivity of the foveal cone photopigments between 400 and $500 \mathrm{~nm}$. Vision Research, 15, $161-171$.

Sterling, P. (1983). Microcircuitry of the cat retina. Annual Review of Neurology, 6, 149-185.

Sterling, P., Freed, M. \& Smith, R. G. (1986). Microcircuitry and functional architecture of the cat retina. Trends in Neuroscience, 9 , 186-192.

Sternheim, C. E. \& Glass, R. A. (1975). Evidence for cone and rod contributions to common "adaptation pools". Vision Research, 15, $277-281$.

Stiles, W. S. (1978). Mechanisms of colour vision. London: Academic Press.

Stockman, A., Sharpc, L. T., Zrenner, E. \& Nordby, K. (1991). Slow and fast pathways in the human rod visual system: ERG and psychophysics. Journal of the Optical Society of America A, 8 , 1657-1665.

Wald, G. (1945). Human vision and the spectrum. Science, 101, $653-658$

Wyszecki, G. \& Stiles, W. S. (1982). Color science (2nd edn). New York: Wiley.

Acknowledgements - This research was supported by the Deutsche Forschungsgemeinschaft, Bonn-Bad Godesberg (SFB 325, B13 and the Heisenberg-Programm) and by the National Science Foundation, Washington (BNS 88-12401 and IBN 92-10046). We are grateful to Steve Buck for helpful comments, and also to the reviewers, whose comments were particularly constructive.

Note added in proof $-0.3 \mathrm{log}$ unit should be added to the threshold values plotted in Figs 1, 2, 3, 4, 6, 9, 11, 13 and 14. (The plotted values are time-averaged intensities rather than amplitudes.) 